\title{
Modelling the field behaviour of a granular expansive barrier
}

\author{
Eduardo Alonso *, Christian Hoffmann \\ Department of Geotechnical Engineering and Geosciences, Universitat Politècnica de Catalunya, cl Jordi Girona 1-3, Campus Norte, \\ Edificio D-2, 08034 Barcelona, Spain
}

Received 25 April 2005; received in revised form 10 April 2006; accepted 26 April 2006

Available online 23 October 2006

\begin{abstract}
The large scale "Engineered Barrier" (EB) experiment, performed at the Mont Terri Underground Laboratory is described. A coupled hydromechanical model is then used to simulate the test performance. Constitutive parameters for the bentonite granular backfill are based on experimental work described in a companion paper. An elastoplastic model describes the granular fill, while the host rock is simulated by a damage model. Predictions of EDZ development around the tunnel are compared with some indirect measurements. Calculated evolutions of relative humidity and stresses within the buffer are compared with sensor records. Good agreement was found for the bentonite blocks supporting the canister. The granular expansive fill exhibit a more irregular behavior. Calculated displacements of the canister agree in absolute terms with actual measurements.
\end{abstract}

(C) 2006 Elsevier Ltd. All rights reserved.

Keywords: Case history; Hydro-mechanical model; Waste disposal; Bentonite; Pellets; Elastoplastic constitutive model; Excavation disturbed zone

\section{Introduction}

The engineered barrier (EB) experiment (AITEMIN, 2001) was designed to demonstrate the feasibility of granular expansive barriers. The proposed concept envisages placing the nuclear canister in a horizontal drift and using a combination of two bentonite materials for filling the empty space surrounding the canister. A lower bed made of compacted bentonite blocks is first constructed to emplace the canister and then a bentonite pelletized material fills the remaining space. Both sealing materials, blocks and pellets, are made of the same FEbEX bentonite (ENRESA, 2000).

The aim of crushed pellets in the upper part of the barrier is to avoid the difficulties of barrier emplacement of alternative concepts previously tested based on block assemblies, as in FEBEX. Since pellets can be projected into the drift from some distance by mechanical means, a

\footnotetext{
* Corresponding author. Fax: +34934017251.

E-mail address: eduardo.alonso@upc.edu (E. Alonso).
}

simpler emplacement operation is possible. Some aspects, which appear to be solved with this type of emplacement technique, are that the emplacement of the pellets can be automated, the upper remaining gap usually found when working with full bentonite block barriers can be minimized, a better contact between the canister and the backfill material is achieved and the emplacement of the canister becomes more accurate.

The full scale demonstration test was performed in the underground laboratory of Mont Terri. The rock hosting the experiment, Opalinus clay, is an impervious clay shale and therefore natural hydration was incapable of providing significant barrier saturation in a reasonable time (say two years). As a result, a forced hydration system was designed and built in order to keep test duration within reasonable times. Field instruments were emplaced to monitor the evolution of the different variables of the experiment (buffer and host rock).

The laboratory experimental program was performed in order to gain insight into the hydro-mechanical response of the material and to derive the material parameters for modelling purposes. 
In order to provide a unified interpretation to the fundamental aspects of the observed response a numerical modelling of the real scale test was performed. The numerical simulation followed the sequence of the in situ test installation and hydration.

Preliminary calculations were first performed to assist in the definition and selection of some system components such as the layout of monitoring instruments and the hydration system. In these calculations, an initial estimation of properties and parameters for the bentonite pellets was done based on previous knowledge of compacted bentonite behaviour. In a second stage, a modelling was based on parameters derived from tests performed on pellet mixtures. The host rock and the fill were described by means of elasto-plastic models (a modified version of the Barcelona Basic Model (BBM), Alonso et al., 1990). The rock model include a damage criteria which was useful to predict the extent of the excavation disturbed zone, which could be compared with in situ determinations based on geophysical procedures and local determinations of permeability (BGR, D18 EB Project, 2003).

The aim of the paper is to describe the modelling performed, placing special emphasis in the comparison between the data provided by the field instruments and the calculations. It has provided a good understanding of the evolution of barrier saturation and of the development of the swelling pressure. The paper is organized as follows: first, the background and objectives of the numerical analysis are presented. Then description of the full scale test is given considering the tunnel construction, material emplacement and wetting procedure. The numerical modelling is later described in some detail. Finally, computed results and the observed behaviour are compared, and some conclusions are derived. It should be emphasized that the EB experiment is a rather unique test within the set of large scale demonstration experiments being performed in the recent years. Uncertainties existed on the feasibility of the test and also on the capabilities of state of the art hydromechanical models to reproduce the actual fill behaviour.

\section{Description of the in situ test}

\subsection{Tunnel construction and EDZ measurements}

A general view of the different galleries of the laboratory where the "EB" test is located is given in Fig. 1. The
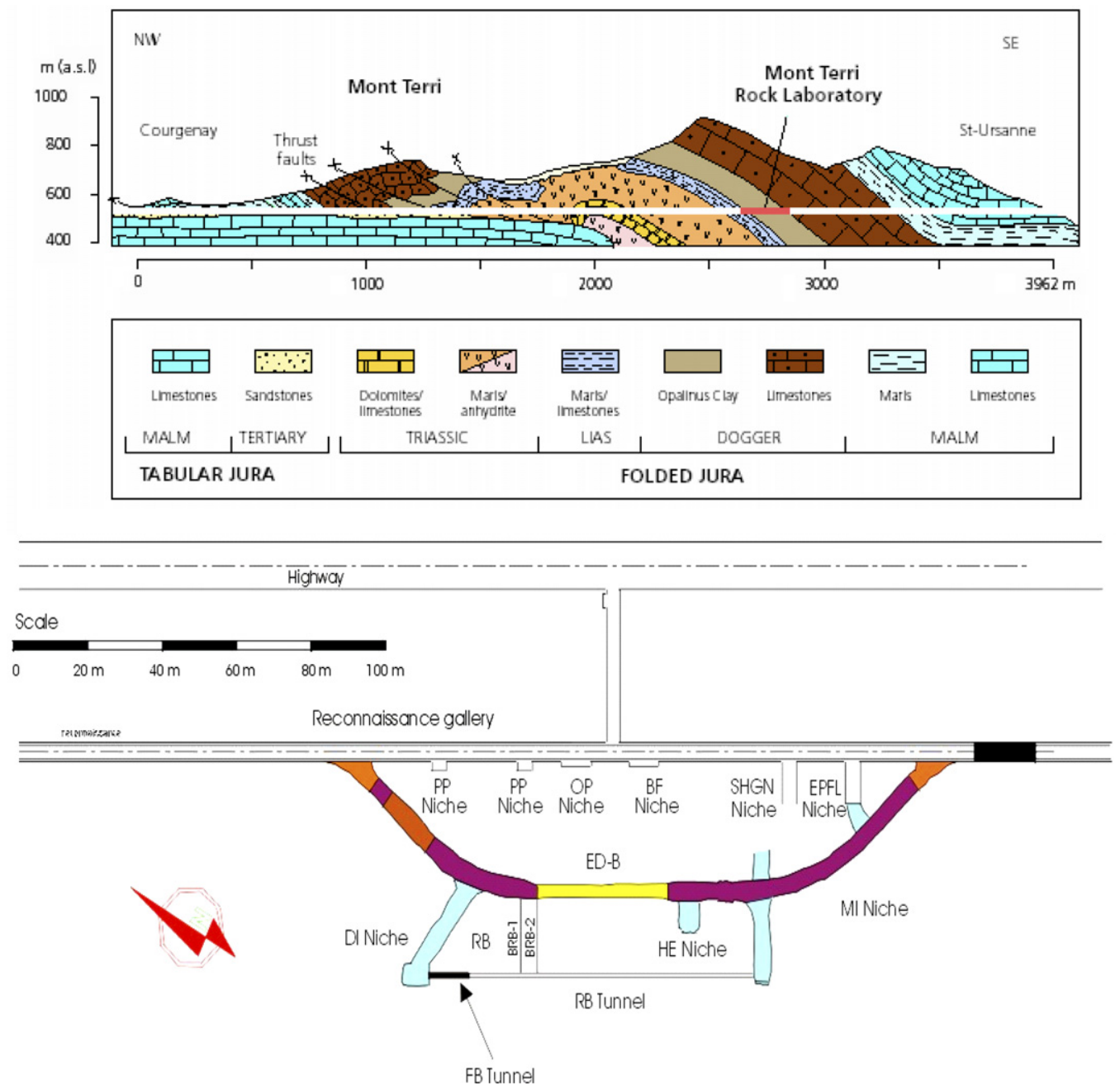

Fig. 1. Geological cross section of the Mont Terri motorway tunnel. Underground laboratory and EB tunnel location (adapted from Bossart et al., 2002). 


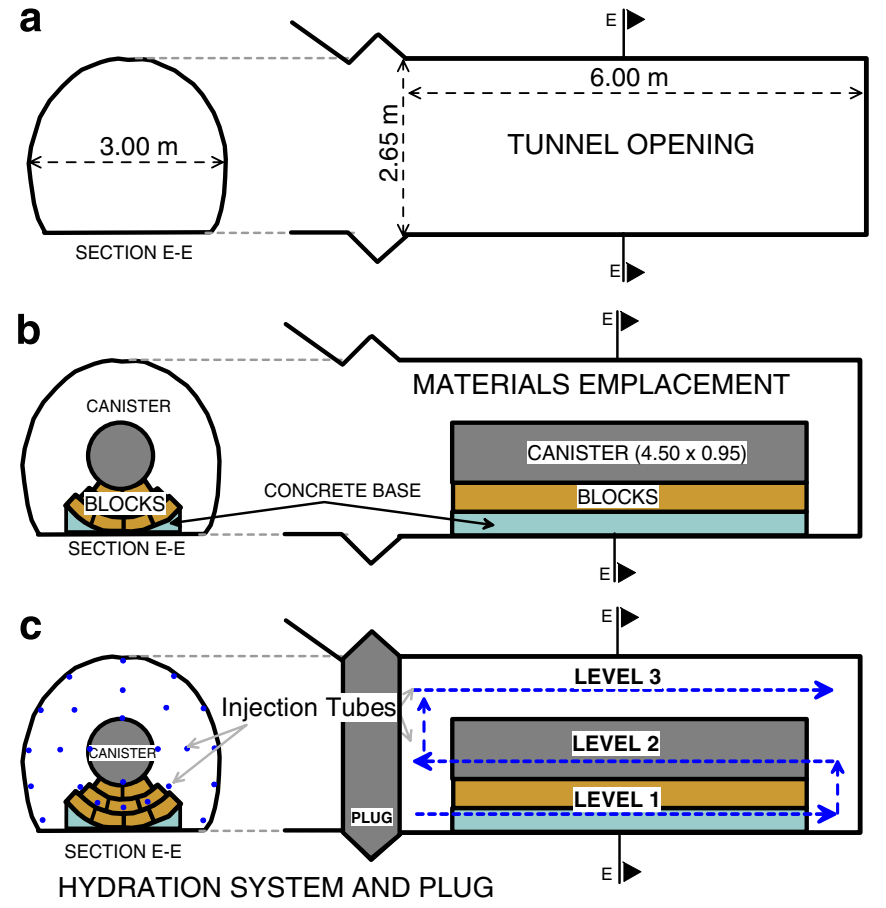

Fig. 2. (a) Tunnel opening, (b) concrete base construction and blocks and canister emplacement and (c) hydration system and concrete plug construction.

geometry and characteristics of the "EB" niche are presented in Fig. 2a (Bossart et al., 2002). The tunnel has a horseshoe cross section. It is $3.00 \mathrm{~m}$ wide and $2.65 \mathrm{~m}$ high and it has a total length of $6.00 \mathrm{~m}$.

The excavation of underground openings generally causes damage to the rock near the wall. This damaged zone (excavation damaged zone (EDZ)) will be considered in the analysis and its role in the interaction between the buffer materials and the host rock will be examined. In order to obtain some information about the EDZ of the excavation performed, a combination of seismic, geoelectric and hydraulic measurements were performed by BGR (2003), once the tunnel excavation was completed.

\subsection{Material emplacement and concrete plug}

Once the "EB" niche was excavated and prior to the installation of the granular mixture other activities have to be completed:

- Construction of a rounded concrete base to hold the bed of "FEBEX" bentonite blocks. The bentonite blocks were manually emplaced as well as the metallic canister simulating the waste, Fig. $2 b$.

- Installation of the sensors, instrumentation system and construction of the artificial hydration system, Fig. 2c.

- Construction of the retaining wall (plug) which isolates the test from the access tunnel, Fig. 2c.

An access window was left open in the plug in order to allow the emplacement of the granular fill. The granular mixture was mechanically emplaced by means of an auger, which went into the "EB" niche through the access window, Fig. 3. The material emplacement operation started from the back and ended at the front of the tunnel. Material compaction was obtained by gravity fall. Because of the presence of the different components of the hydration system, very little space was available for the auger to move. This fact results in a lower than expected dry density value for the granular material. According to reported data (NAGRA, 2003), the average dry density obtained was about $1.36 \mathrm{Mg} / \mathrm{m}^{3}$. This value is about $3 \%$ lower than the value obtained in previous emplacement trials performed in a one-to-one scale wooden tunnel, NAGRA (2003). In additional tests performed prior to the test emplacement, the average drop density of the fill was $1.46 \mathrm{Mg} / \mathrm{m}^{3}$, with maximum and minimum values of 1.52 and $1.28 \mathrm{Mg} / \mathrm{m}^{3}$, respectively (NAGRA, 2003). Therefore, a significant heterogeneity in the spatial distribution of dry density was expected in the "EB" experiment. The transient collapse effects observed during wetting, reported later, are an indication of this heterogeneity.

Once the granular material was emplaced, the access window was closed and the hydration phase was prepared.
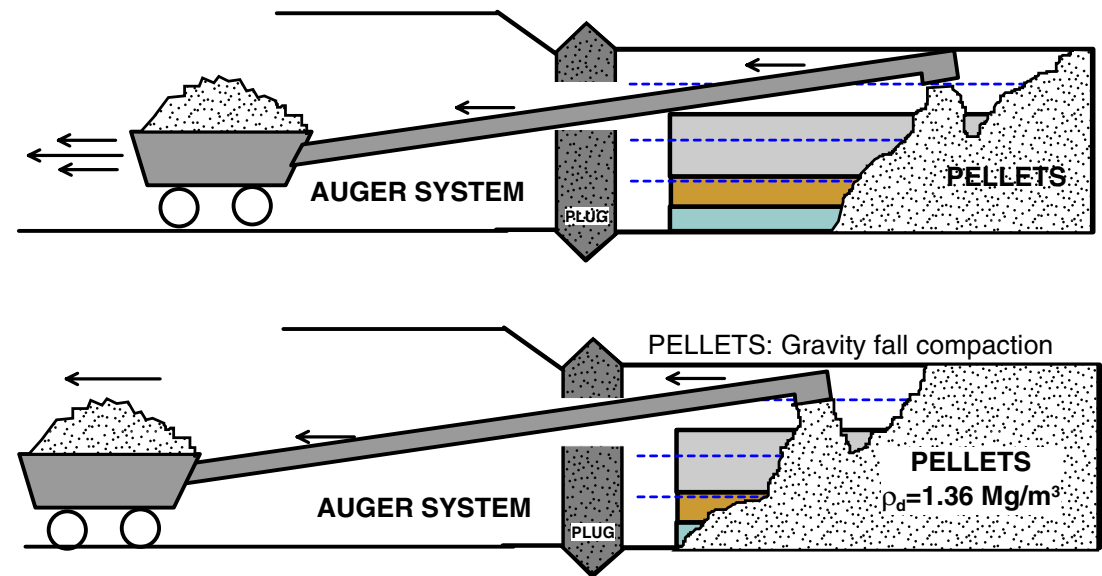

Fig. 3. Emplacement of bentonite pellet based material. Pellet material is compacted by gravity fall. 


\subsection{Hydration system}

Due to the small amount of free water available in the Opalinus clay shale formation, an artificial hydration system was needed in order to get the buffer saturated within reasonable testing times. The selection and definition of the characteristics of the hydration system were done based on the preliminary simulations. In these simulations, material laws and parameters used for the host rock and the bentonite blocks were taken from previous studies but no information concerning the bentonite crushed pellets was available at the beginning of the project. For this reason, parameters for the granular fill had to be estimated based on existing hydro-mechanical characterizations available for FEBEX blocks. Parameters for the pellet mixture were scaled using known properties of the compacted block material and the dry density as the scaling parameter.

Preliminary simulations indicated that in order to obtain full saturation within the expected times, three layers of hydration points had to be emplaced. A distance of $0.15 \mathrm{~m}$ between wetting tubes was suggested.

Based on these recommendations, the hydration system was designed and built. 37 Injection tubes were arranged in a three layer configuration. Additionally, a pervious mat was used to cover the tubes, canister and the bentonite blocks in order to favour the water distribution in these zones. A cross section of the tunnel showing a schematic view of the hydration system is illustrated in Fig. 4a. Tubes were connected in such a way that the water flows into buffer materials from the floor to the roof of the excavation as indicated in Fig. 4b. In order to favour the injection process and to allow the air existing in the buffer and in the water injection system to escape, the upper tube was connected to the access gallery.

\subsection{Instrumentation}

A number of sensors were placed at different tunnel sections, Fig. 5. relative humidity sensors (rock and buffer), displacement sensors (canister and rock), piezometers (rock) and total pressure cells (buffer) were placed at different radial distances.

The movement of the canister was monitored by measuring the horizontal and vertical displacements of two fixed points of the canister by means of extensometers located in sections A1 and A2, Fig. 6a.

The position of the relative humidity sensors in the cross sections B1 and B2 is shown in Fig. 6b. Swelling pressure cells were placed in sections $\mathrm{E}$ at the contacts between the buffer and the canister and between the tunnel wall and the buffer as indicated in Fig. 6c. Plotted in Fig. 7 are the relative humidity sensors placed in sections A1 and A2. Their measurements will be later compared with calculations.

\subsection{Testing procedure}

Due to the high interconnectivity existing in the granular fill because of the large inter-pellet voids, high initial water injection rates were expected at the beginning of the hydration phase. After this initial period, the bentonite pellets begin to swell filling the inter-pellet voids, blocking the incoming water and making the water permeability to decrease several orders of magnitude. This phenomenon was observed on different infiltration tests performed during the experimental program.

In view of this behaviour, it was decided to apply a high injection pressure, with the purpose of maximizing the initial water intake and of reducing the expected saturation time.

During the first part of the hydration phase, water pressure was held constant and a volume of $6.7 \mathrm{~m}^{3}$ of water was injected during the first two days. Immediately afterwards the water injection was stopped because some of the injected water was observed to be coming out of the tunnel. These loses were attributed to sealing problems in the concrete plug or, alternatively, to the presence of conductive joints in the rock. No water was injected for 120 days.

a
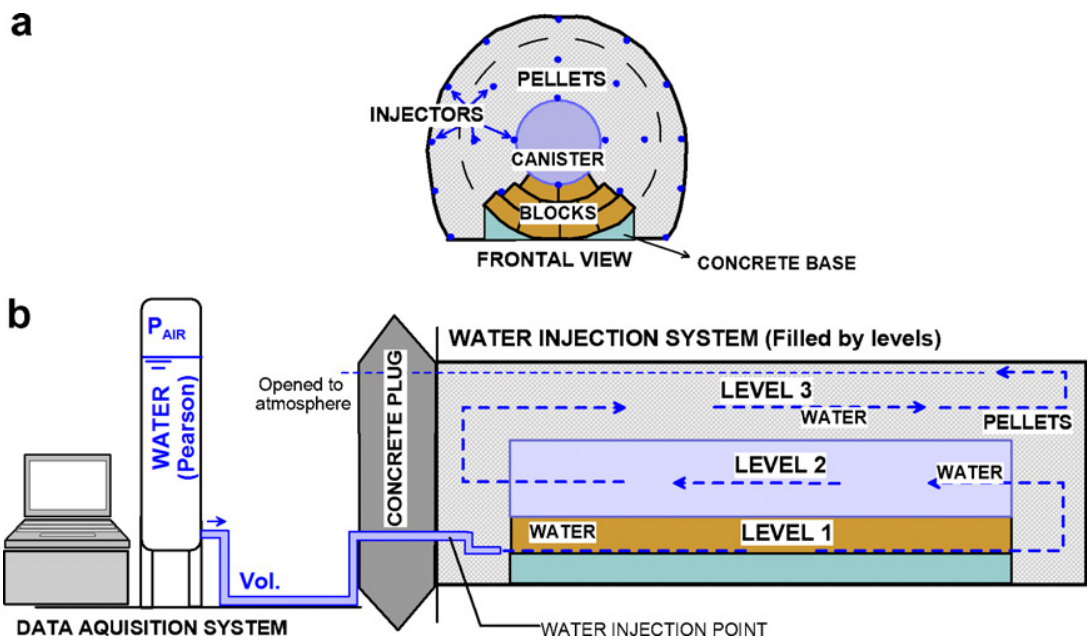

Fig. 4. Hydration system. 


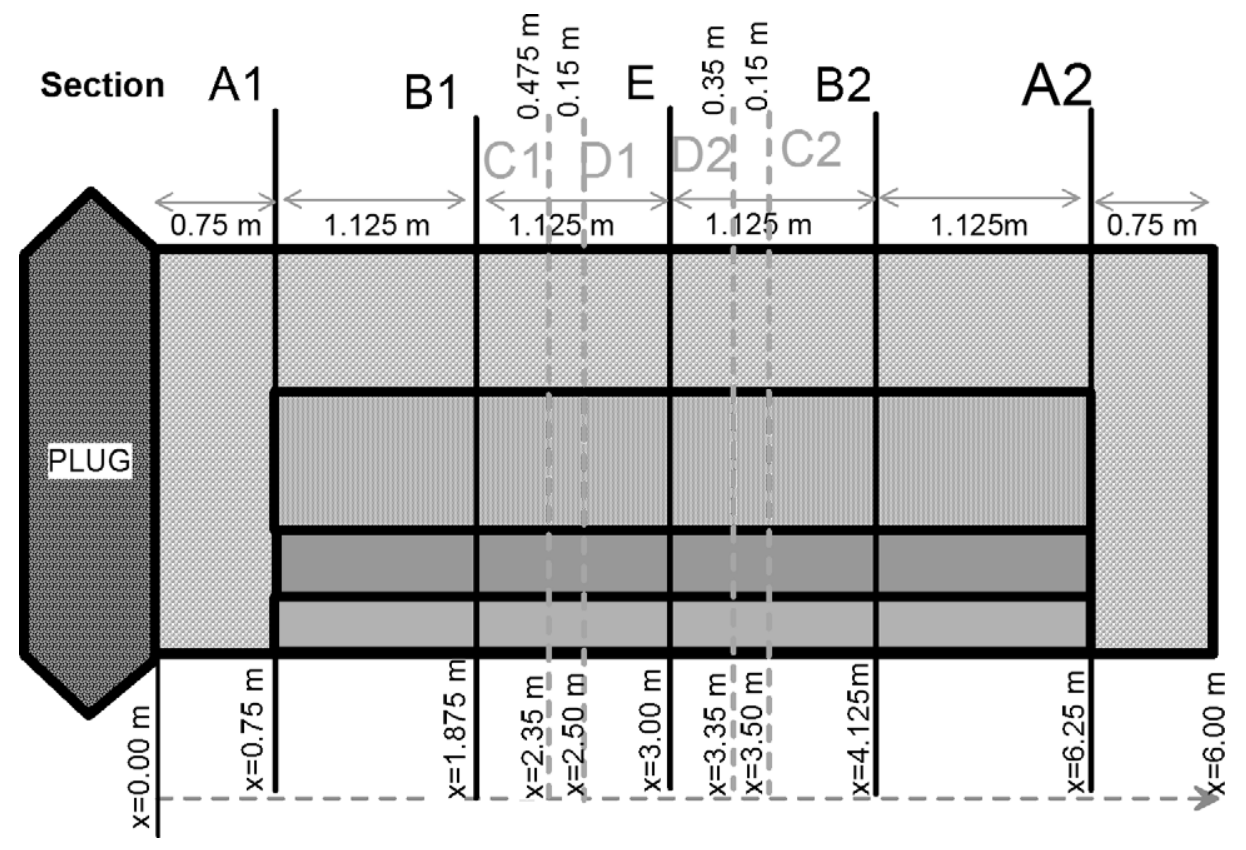

Fig. 5. Different tunnel sections for the installation of sensors.

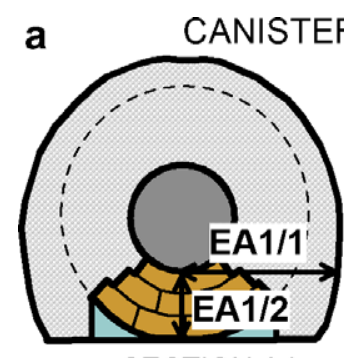

SECTION A1
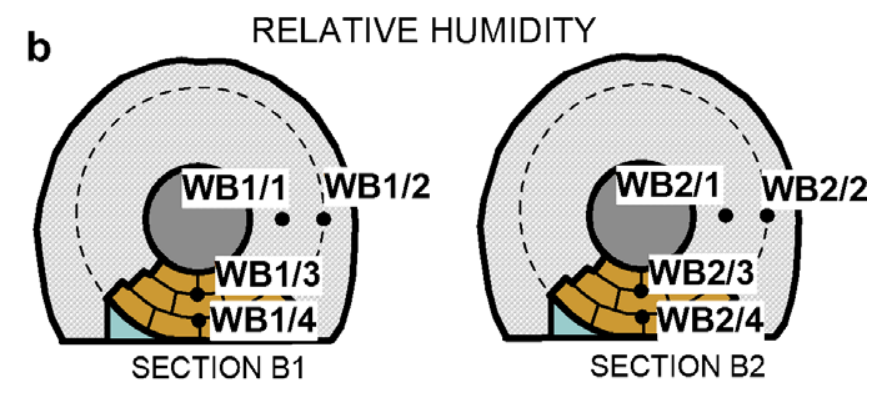

C

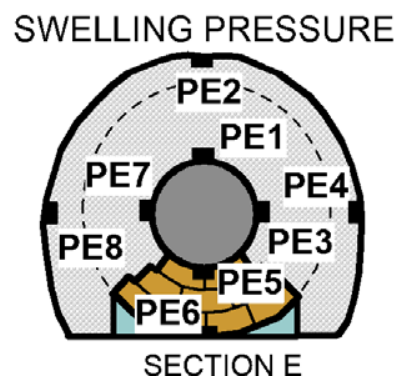

Fig. 6. Location of the different sensors in the buffer. EA: Extensometers; WB: Relative humidity; PE: Total pressure.

After this period water injection was resumed and a total flux rate criterion was adopted to control the injection. A volume of 251 per day was injected, in two stages (12.51, twice a day, in a ten minute interval). Fig. 8 shows the history of water injection during the period May 2002-January 2004.

\section{Numerical modelling}

For modelling purposes, the problem was divided in two parts. First the tunnel construction was simulated and then, a hydration phase was considered. During tunnel construction, the initial stress and water pressure fields for later steps were obtained and some analysis regarding the evolution of the EDZ was performed. After this initial step, the buffer hydration phase was simulated and a comparison between computed results and observed behaviour was done.

\subsection{Geometry, initial and boundary conditions}

\subsubsection{Initial state (Fig. 9a)}

The two dimensional plain strain domain considered for the "start" configuration of the problem has $80 \mathrm{~m}$ wide and $80 \mathrm{~m}$ height (with a central plane of symmetry). This implies a minimum distance of $25 R_{\text {Tunnel }}$ from the centre of the EB niche to the boundaries $\left(R_{\text {Tunnel }}\right.$ is the average tunnel radius).

An anisotropic stress field with vertical and horizontal stress values of 6.0 and $4.8 \mathrm{MPa}$, respectively and a constant water pressure field of $1.0 \mathrm{MPa}$ was the assumed initial state. These values were approximated from data available for the Mont Terri site (Bossart et al., 2002). The following boundary conditions were assumed in calculations: 

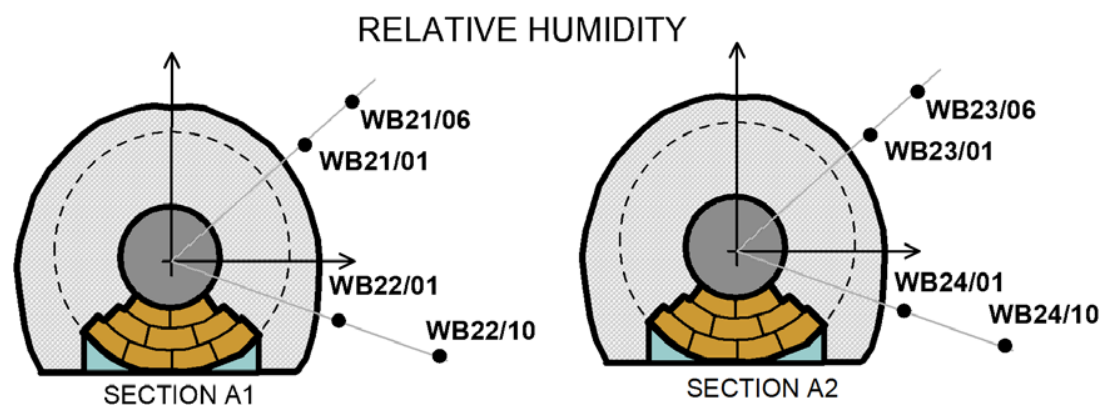

Fig. 7. Location of the different sensors in the rock. WB: Relative humidity sensors.

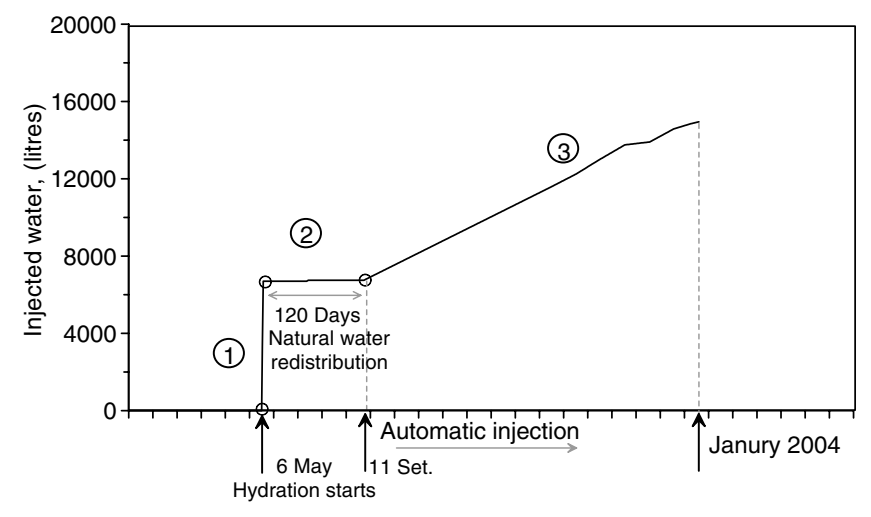

Fig. 8. Water injection history.

- no horizontal displacement was allowed along vertical boundaries.

- no vertical displacement was permitted along the lower horizontal boundary of the considered domain.

- a compressive vertical stress of $6.0 \mathrm{MPa}$ was applied along the upper horizontal boundary.

- a constant water pressure value of 1.0 MPa was applied along the upper and lower horizontal boundaries of the domain and along the vertical boundary situated far from the tunnel opening.

\subsubsection{Tunnel opening (Fig. 9b)}

Once the initial state configuration was defined, the tunnel construction was simulated. This part of the analysis provides the stress and water pressure fields, prevailing at the time of buffer emplacement (160 days after tunnel opening in this case) and the expected development of damaged zones around the tunnel (EDZ). A relative humidity, $\mathrm{RH}=93 \%$, equivalent to a suction of $10 \mathrm{MPa}$ was adopted as a boundary condition on the tunnel surface.

\subsubsection{Buffer emplacement and hydration phase (Fig. 9c)}

Once the tunnel is opened the buffer materials are emplaced and the hydration phase starts. The initial water pressure and stress fields for this stage correspond to the end of the construction period (160 days). The initial, boundary conditions, section shape and distribution of materials for this step are illustrated in Fig. 9c. Suction val- ues of $300 \mathrm{MPa}$ and $150 \mathrm{MPa}$ were assumed as initial values for the bentonite pellets and blocks, respectively. An isotropic state of confining stress of $0.3 \mathrm{MPa}$ was selected as the initial state for both bentonite materials.

\subsection{Constitutive models}

The host rock and the bentonite fill materials were described by means of elasto-plastic models. Perfectly elastic models were utilized to represent the concrete base, the metallic canister and the geotextile material.

\subsubsection{Host rock}

Opalinus clay. The Opalinus clay is a claystone that is idealized as a composite material, made of a clay matrix interlocked by bonds. Pearson et al. (2003) presented a comprehensive description of the Opalinus clay. Vaunat and Gens (2003) formulated a constitutive model for bonded materials outlined below. In this model, bonds response follows a damage model proposed by Carol et al. (2001), whereas the clay matrix was described by means of an elastoplastic model based on a Hoek and Brown failure criterion.

A Terzaghi effective stress principle was adopted. Volumetric changes due to changes in suction are incorporated via the effective stress law. The elastic component of deformations follows a classical linear isotropic model $(E$ : Young's modulus; $v$ : Poisson's ratio). The Young's modulus is described in terms of the clay matrix and bond properties as follows:

$E=(1+\chi) E_{\mathrm{M}}(1+\chi)+\chi E_{\mathrm{b}} \chi$

where $E_{\mathrm{M}}$ and $E_{\mathrm{b}}$ represents the elastic moduli of the clay matrix and bonds, respectively. The parameter $\chi$ takes into consideration the progressive degradation of the rock.

Within the elastic regime and assuming the same Poisson's ratio $v$ for bond and clay matrix, conditions of local stress equilibrium for triaxial stress state takes the form:

$p=\left(1+\chi+\frac{\chi^{2}}{1+\chi} \frac{E_{\mathrm{b}}}{E_{\mathrm{M}}}\right) p^{M}$ 


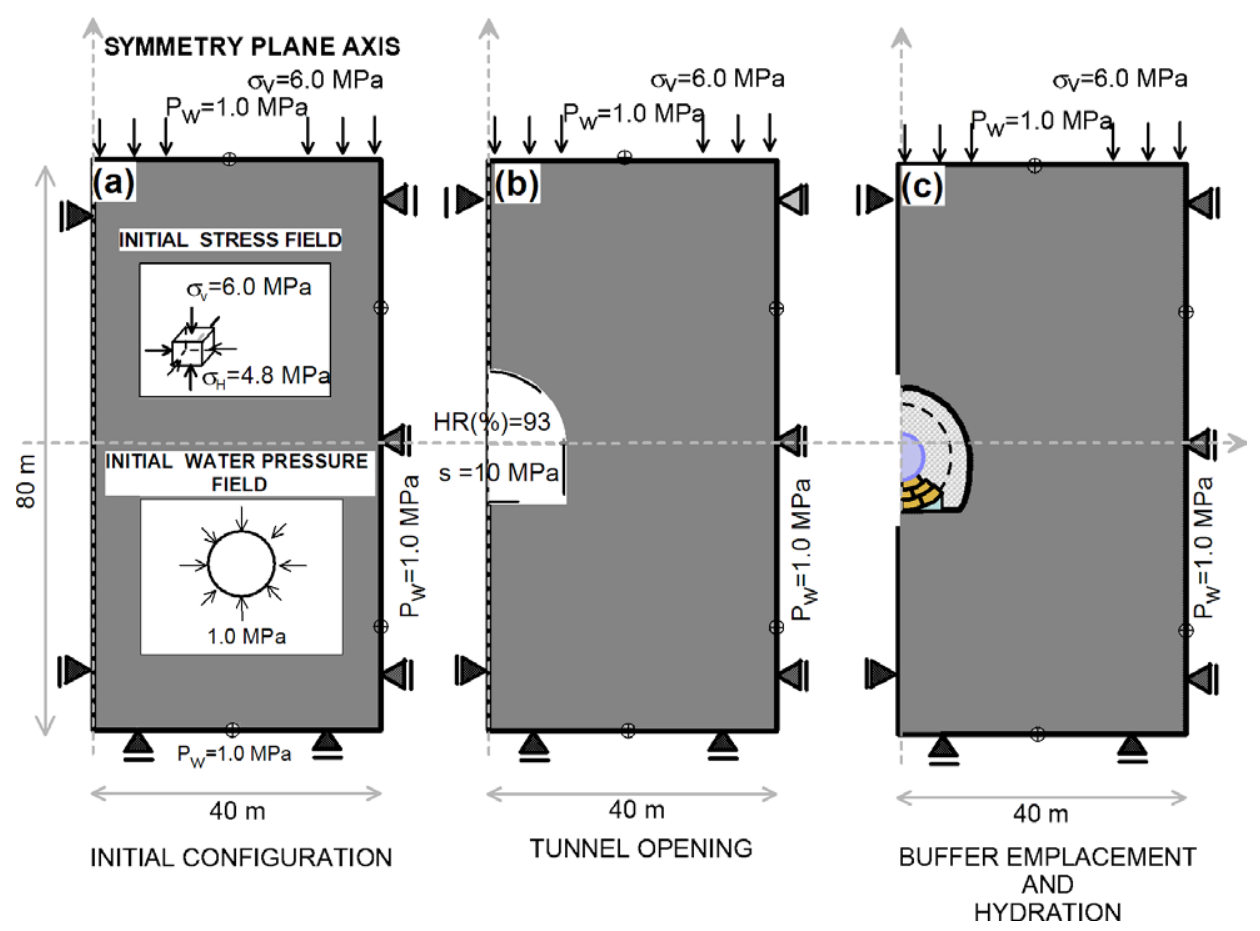

Fig. 9. (a) Initial configuration, (b) tunnel opening and (c) buffer emplacement and hydration.

and

$q=\left(1+\chi+\frac{\chi^{2}}{1+\chi} \frac{E_{\mathrm{b}}}{E_{\mathrm{M}}}\right) q^{M}$

where $p$ and $q$ are the external net mean and deviatoric stresses and $p^{M}$ and $q^{M}$ are the equivalent values within the clay matrix.

At failure the Hoek and Brown criterion is expressed as a function of external stresses as follows:

$\frac{q^{2}}{R_{\mathrm{c}}}-\frac{m}{3} q-m\left(p+p_{\mathrm{t}}\right)-R_{c}=0$

where

$R_{\mathrm{c}}=R_{\mathrm{cM}}\left(1+\chi+\frac{\chi^{2}}{1+\chi} \frac{E_{\mathrm{b}}}{E_{\mathrm{M}}}\right)=R_{\mathrm{cM}} \frac{E}{E_{\mathrm{M}}}$

and

$p_{\mathrm{t}}=\frac{R_{\mathrm{c}}}{m}=p_{\mathrm{tM}}\left(1+\chi+\frac{\chi^{2}}{1+\chi} \frac{E_{\mathrm{b}}}{E_{\mathrm{M}}}\right)=p_{\mathrm{tM}} \frac{E}{E_{\mathrm{M}}}$

where $R_{\mathrm{cM}}$ is the clay matrix uniaxial strength and $p_{\mathrm{tM}}$ the clay matrix tensile strength. $m$ represents the ratio between the compressive and tensile strength of the material.

Parameter $\chi$ varies with the degradation of elastic moduli according to the following expression:

$\chi=\chi_{0} e^{-L / 2}$

where $\chi_{0}$ represents the state of the rock when bonds are intact and the parameter $L$ describe its evolution. The $L$ factor introduces the decay in elastic moduli often observed in triaxial testing of rock specimens. Certainly, other mea- sures of damage could be devised but the major difficulty probably lies in getting an adequate factor for the rock massif itself. $L$ provides one of the simplest choices. This parameter takes a zero value in the undamaged state and an infinite value in the fully damaged state. $L$ is simply related to the degradation of Young's modulus, following the expression $L=\ln \left(E_{0} / E\right)$ where $E_{0}$ and $E$ are limiting values for the undamaged and damaged Young's modulus, respectively. Table 1 summarizes the main characteristics of the model.

Fig. 10 shows the yield envelopes considered for triaxial stress conditions. Both the yield criterion for the composite material and the degradation law surfaces for the bonds are represented in the same plot with coincident axis. The horizontal axis represents the total effective net mean stress for the composite material $(p)$ and the effective net mean stress corresponding to the bonds $\left(p_{\mathrm{b}}\right)$. In the vertical axis $(q)$ and $\left(q_{\mathrm{b}}\right)$ represent the deviatoric stresses. In this figure yield and damage surfaces indicated as $\chi_{0}$ corresponds to the

Table 1

Equations defining the constitutive law of the Opalinus clay

\begin{tabular}{lll}
\hline Law & Description & Equation \\
\hline Elastic & Linear elastic & $E^{2}=(1+\chi) E_{\mathrm{M}}(1+\chi)+\chi E_{\mathrm{b}} \chi$ and $v$ \\
Yield & Hoek and Brown & $\frac{q^{2}}{R_{\mathrm{c}}}-\frac{m}{3} q-m\left(p+p_{\mathrm{t}}\right)-R_{\mathrm{c}}=0$ \\
$\quad$ surface & criterion & $R_{\mathrm{c}}=(1+\chi) \cdot R_{\mathrm{cM}}$ and $m=$ constant \\
$\begin{array}{l}\text { Flow rule } \\
\text { Degradation }\end{array}$ & Associated & Exponential \\
$\quad$ law & & $\chi=\chi_{0} \mathrm{e}^{-L / 2}$ \\
$\begin{array}{c}\text { Damage } \\
\text { locus }\end{array}$ & Ellipse & $F_{\mathrm{d}}=u_{\mathrm{b}}-r(L) \leqslant 0 \quad$ where \\
\hline
\end{tabular}




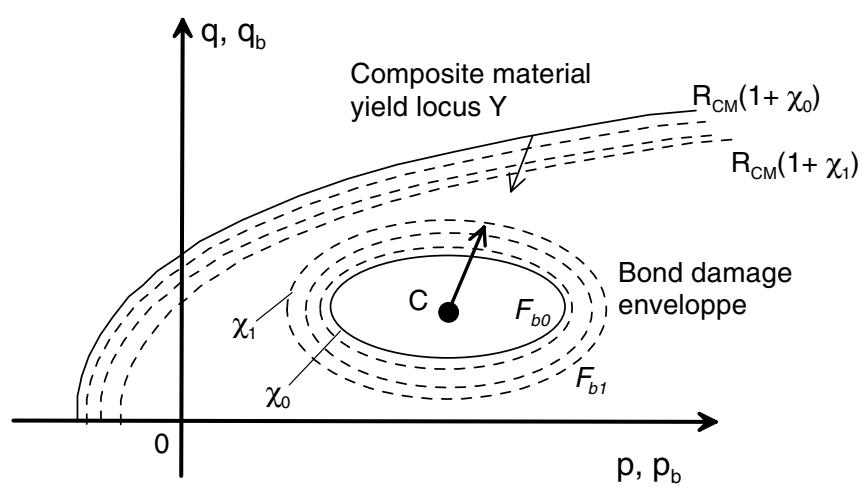

Fig. 10. Yield surfaces for the constitutive model considered for the host rock (Opalinus clay) under triaxial conditions.

Table 2

Parameters used for Opalinus clay (Muñoz et al., 2004)

\begin{tabular}{lllllll}
\hline $\begin{array}{l}\text { Young's modulus } \\
(\mathrm{MPa})\end{array}$ & $\begin{array}{l}\text { Poisson } \\
v\end{array}$ & $\begin{array}{l}R_{\mathrm{c}} \\
(\mathrm{MPa})\end{array}$ & $m$ & $\chi_{0}$ & $\begin{array}{l}r_{0} \\
(\mathrm{MPa})\end{array}$ & $\begin{array}{l}r_{1} \\
(\mathrm{MPa})\end{array}$ \\
\hline 7000 & 0.27 & 16 & 8 & 2 & 0.01 & 0.105 \\
\hline
\end{tabular}

undamaged state whereas the surfaces indicated with $\chi_{1}$ corresponds to a certain damage state.

Parameters, given in Table 2, were determined based on laboratory data. $E$, $v$, and $R_{\mathrm{cM}}$, were assessed from tests on intact rock samples. A set of values for $R_{\mathrm{c}}$ were obtained from tests performed on samples with certain damage. Then, values for $\chi$ were derived from reference in order to give the upper and lower envelopes for all measured shear strengths. $m$ was defined from data on the tensile strength of the material. $E_{\mathrm{M}}$ and $E_{\mathrm{b}}$ were derived from values of $E$ and $\chi_{0}$, assuming that $E_{\mathrm{b}}=E$ in the undamaged state $\left(\chi=\chi_{0}\right)$.

The initial damage locus (indicated as $F_{\mathrm{b} 0}$ in Fig. 10) for triaxial conditions is an ellipse with major and minor principal axis equal to $\sqrt{K_{\mathrm{b}} r(L)}$ and $\sqrt{G_{\mathrm{b}} r(L)}$, where $K_{\mathrm{b}}$ and $G_{\mathrm{b}}$ are the secant bulk and shear moduli of the bonds. $r(L)$ is the damage evolution law and was derived from value of stiffness degradation obtained from cyclic loading-unloading triaxial tests.

\subsubsection{Buffer materials}

The BBM elastoplastic model (Alonso et al., 1990) described compacted blocks and the granular pellet fill. Details to the model, parameter description and its application to the modelling of laboratory tests is given in Hoffmann et al. (2006).

\subsection{Model parameters}

Parameter values for the bentonite blocks were approximated from data reported in the FEBEX final report (2000). For the bentonite pellets, the experimental information discussed in Hoffmann et al. (2006) provided the necessary data. The relevant characteristics of the pellet mixtures tested in the laboratory are summarized as follows:

- Pellet mixtures exhibit a remarkable multi model structure. In general, two groups of voids can be defined. The largest size is associated with the inter-pellet voids (macropores) whereas the smaller sizes (micropores associated with the inter and intra clay aggregates) are found inside the highly compacted pellets. The largest pores disappear when the dry density of the mixture increases but also when the mixture is wetted.

- The hydraulic response of the material when it is first exposed to a water injection is characterized by the high connectivity of the network of the inter-pellet voids. Therefore, high inflow rates are expected. However, as the dense pellets hydrate, at the expense of the free circulating water they swell, fill the macropores and the permeability decreases several orders of magnitude. This behaviour is illustrated in Fig. 11, which shows the evolution of the apparent permeability with the degree of saturation observed during an infiltration test (Hoffmann et al., 2006). The relationship given in Fig. 11 is useful for modelling purposes. It provides the change in the intrinsic permeability and relates it to the degree of saturation, making if possible to conduct realistic flow analysis with limited effort.

- The outlined behaviour at microscopic level explains the constitutive response observed when specimens are wetted. If the water infiltrates fast into the sample, the interpellet voids became saturated and the intergranular forces are reduced. Then, a global collapse of the granular structure could take place. It depends on the stress level existing in the fill. Later, as the expansive pellets hydrate, a swelling response is measured. The macroscopically observed behaviour is the result of both mechanisms and their interaction. In particular, different rates of wetting lead to different mechanical responses.

For modelling purposes, the pellet mixture has been characterized by a modified version of the BBM, which incorporates the main features of the behaviour outlined. Experimental data was available for a range of dry

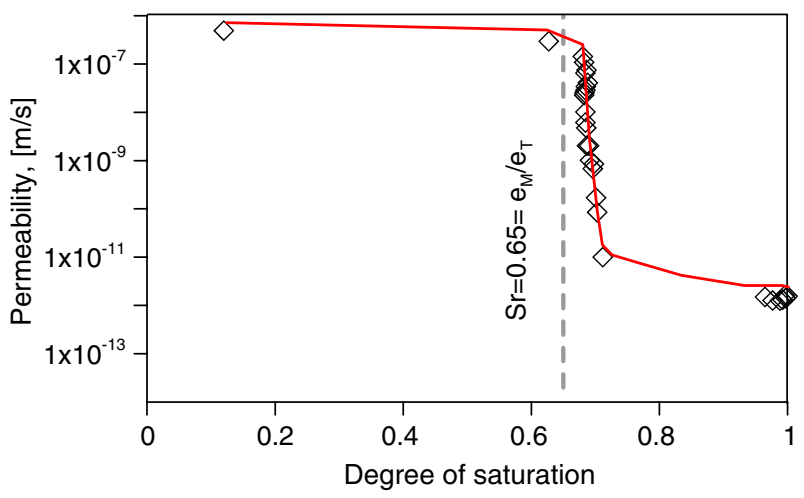

Fig. 11. Evolution of the permeability of the bentonite pellet material with the degree of saturation observed during an infiltration test. 
densities. Parameters were interpolated for the average in situ dry density $\left(\rho_{\mathrm{d}}=1.36 \mathrm{Mg} / \mathrm{m}^{3}\right)$.

Fig. 11 suggests that a suitable simplification for the variation of the permeability with saturation is to accept a discrete "two-permeability" model. During the fast injection of water (first two days) a high permeability $\left(k=10^{-8} \mathrm{~m} / \mathrm{s}\right)$ was assumed. The subsequent redistribution period (Fig. 8) leads to a major reduction in permeability $\left(k=10^{-12} \mathrm{~m} / \mathrm{s}\right)$. In fact, the average degree of saturation computed after the initial volume of water was injected is $\mathrm{Sr}=0.8$, well within the range of "low permeability" shown in Fig. 11.

A dependence of the elastic compressibility $\kappa_{\mathrm{i}}$ with the current suction was introduced (Table 3). The swelling behaviour was modelled through the elastic compressibility coefficient, $\kappa_{\mathrm{s}}$. To be realistic, $\kappa_{\mathrm{s}}$ was made dependent on the net mean stress and suction (see Table 4).

The set of constitutive parameters for the modified BBM used in computations are given in Tables 3-6 for the blocks and the pellets mixture. The deviatoric behaviour was characterized by a common critical state slope $(M=1.5)$ and a value $k=0.1$.

In order to reproduce the hydraulic response of the bentonite pellet material, a "two-permeability" model with intrinsic permeability values ranging from $K\left[m^{2}\right]=1 \times$ $10^{-15}$ to $K\left[m^{2}\right]=1 \times 10^{-20}$ was adopted. In case of the bentonite blocks, an intrinsic permeability value of $K\left[\mathrm{~m}^{2}\right]=$ $1 \times 10^{-21}$ was used during all the hydration phase.

Table 3

Parameter values for the $\kappa_{\mathrm{i}}(s)=\kappa_{\mathrm{io}} \cdot\left(1+\alpha_{\mathrm{i}} * s\right)$ relationship

\begin{tabular}{lcc}
\hline Parameter & Pellets & Blocks \\
\hline$\kappa_{\mathrm{io}}$ & 0.06 & 0.02 \\
$\alpha_{\mathrm{i}}\left(\mathrm{MPa}^{-1}\right)$ & -0.0015 & -0.003 \\
\hline
\end{tabular}

Table 4

Parameter values for the $\kappa_{\mathrm{s}}=\kappa_{\mathrm{s} 0}\left[1+\alpha_{\mathrm{sp}} \operatorname{Ln}\left(\frac{p}{p_{\text {ref }}}\right)\right] \mathrm{e}^{\alpha_{\mathrm{ss}} s}$ relationship

\begin{tabular}{lcc}
\hline Parameter & Pellets & Blocks \\
\hline$\kappa_{\mathrm{s} 0}$ & 0.03 & 0.052 \\
$\alpha_{\mathrm{sp}}$ & -0.13 & -0.12 \\
$\alpha_{\mathrm{ss}}\left(\mathrm{MPa}^{-1}\right)$ & -0.016 & -0.007 \\
$p_{\text {ref }}(\mathrm{MPa})$ & 0.01 & 0.01 \\
\hline
\end{tabular}

Table 5

Parameter values for the $\lambda(s)=\lambda(0) \cdot\left[r+(1-r) \mathrm{e}^{-\beta s}\right]$ relationship

\begin{tabular}{lll}
\hline Parameter & Pellets & Blocks \\
\hline$\lambda(0)$ & 0.17 & 0.18 \\
$r$ & 0.6 & 0.75 \\
$\beta\left(\mathrm{MPa}^{-1}\right)$ & 0.05 & 0.05 \\
\hline
\end{tabular}

Table 6

Parameter values for the yield surface $p_{0}\left(s, p_{0}^{*}\right)=p^{c}\left(\frac{p_{0}^{*}}{p^{c}}\right)^{\frac{\left(\lambda(0)-\kappa_{\mathrm{i}}\right)}{\left(\lambda(s)-\kappa_{\mathrm{i}}(s)\right)}}$

\begin{tabular}{llc}
\hline Parameter & Pellets & Blocks \\
\hline$p_{0}^{*}(\mathrm{MPa})$ & 1.0 & 14 \\
$p^{c}(\mathrm{MPa})$ & 0.075 & 0.1 \\
\hline
\end{tabular}

\section{Comparison between computed results and observed behaviour}

\subsection{Tunnel construction}

Modelling tunnel construction provided water pressure and stress fields at the end of the excavation. They constitute the initial conditions for the buffer hydration phase. It was also useful to obtain some information about the influence of the excavation damage zone (EDZ) on the barrier performance. In the following section, the evolution of the water pressure and stress field after tunnel opening and during the ventilation period are presented. A sensitivity analysis of the computed rock response is presented. The effect of the tunnel relative humidity $(\mathrm{RH})$ and the rock permeability are explored. A comparison between computed results and measured values is also presented.

\subsubsection{Water pressure and stress fields}

A ventilation period of 160 days was assumed considering a constant relative humidity $(\mathrm{RH})$ value of $93 \%$ inside the tunnel. This value was adopted on the basis of some numerical simulations performed during the heating experiment (HE) performed at Mont Terri (Muñoz et al., 2004). Calculated contours of water pressure in the rock after 160 days of ventilation are illustrated in Fig. 12. Only positive water pressure values are indicated. The water pressure profile in the rock along a horizontal axis $(O x)$ is also plotted. Note that a ring of rock, having negative pore pressure, is calculated around the tunnel. It has an average thickness of $1 \mathrm{~m}$. This is indicated also in Fig. 13a. Total stress fields at the end of the 160 days ventilation period are illustrated in Fig. $13 \mathrm{~b}$ and c. Stress changes along horizontal $(O x)$ and vertical $(O y)$ axes are presented. The circumferential stress reaches a peak a short distance into the rock. It marks the development of the plastic zone, which is a thin layer of rock around the tunnel surface.

In order to analyze the influence of the relative humidity value assumed during the ventilation phase and the intrinsic permeability value adopted for the rock, a sensitivity analysis was performed. Three different cases were considered combining different values as shown in Fig. 14. The RH values were related to suction values by means of the psychometric law given by:

$\Psi=-\frac{\rho_{\mathrm{w}} R T}{M_{\mathrm{w}}} \operatorname{Ln}\left(\frac{u_{\mathrm{v}}}{u_{\mathrm{v} 0}}\right)$

where $\Psi$ is the total suction $(\mathrm{kPa}), \rho_{\mathrm{w}}$ is the water density $\left(\mathrm{m}^{3} / \mathrm{kg}\right), R$ is the universal gas constant $(8.31432 \mathrm{~J} / \mathrm{mol}$ $\mathrm{K}), T$ is the absolute temperature $\left(T=273.16+t^{\circ}\right.$, in $\left.\mathrm{K}\right)$, $\omega_{\mathrm{v}}$ is the molecular mass of water $(18.016 \mathrm{~kg} / \mathrm{mol})$ and $\frac{u_{\mathrm{v}}}{u_{\mathrm{v} 0}}$ gives the relative humidity as the ratio between the partial pressure of water vapour in the air $\left(u_{\mathrm{v}}\right)$ and the partial pressure at saturation $\left(u_{\mathrm{v} 0}\right)$. 


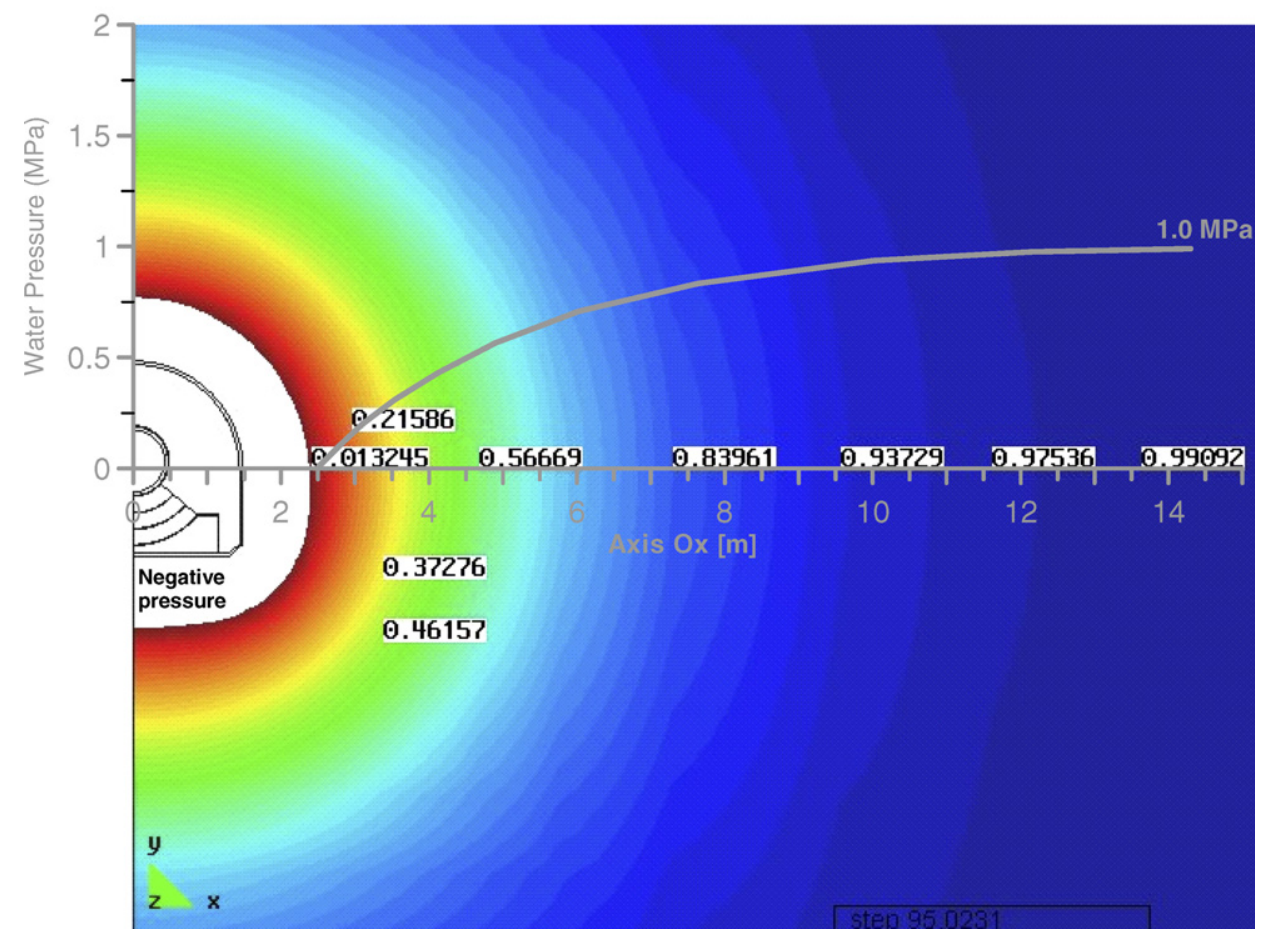

Fig. 12. Water pressure field after 160 days of tunnel opening. A drained zone of about $1 \mathrm{~m}$ thick is calculated.

Suction records based on model predictions and in situ measurements, once the tunnel was opened, during the ventilation period are shown in Figs. 15 and 16 for cross sections A1 and A2. In all the plots the history of injected volume of water after the ventilation period is also indicated as a general reference (the water injection phase is described in more detail later). The two instrumented sections $\mathrm{A} 1$ and $\mathrm{A} 2$ displays almost the same response during this period. According to the values registered in sensors located at a distance of $0.1 \mathrm{~m}$ from the tunnel wall (WB0_01, WB2_01, WB23_01 and WB24_01), the relative humidity value in the rock, near the tunnel wall was about $80 \%$ after almost 100 days of ventilation. This is equivalent to a suction of around $30 \mathrm{MPa}$ which is calculated with the expression (8). On the other hand, sensors located at distances of 0.6 and $1.0 \mathrm{~m}$ from the tunnel wall (WB0_06, WB2_10, WB23_06 and WB24_10) registered a relative humidity value of around $95 \%$, indicating a suction value of $7 \mathrm{MPa}$. The best agreement is found for case $3(89 \%$ $\mathrm{RH}$ in the tunnel and a rock intrinsic permeability of $0.5 \times 10^{-19} \mathrm{~m}^{2}$ ) although some discrepancies remain. It is concluded that the relative humidity value assumed initially for the ventilation period seems to be higher than the in situ value. The plots in Figs. 15 and 16 point out the dominant effect of rock permeability to control the evolution of the $\mathrm{RH}$ inside the rock.

\subsubsection{EDZ development}

Because of the excavation process and the subsequent ventilation phase, a rock damage zone is produced around the tunnel. Tunnel excavation modifies the state of stress existing in the rock and some damage is induced. The damaged zone evolves thereafter because of the suction increase induced during ventilation. An increase in suction increases the effective means stress which in turn causes some additional bond damage.

The development of the EDZ is presented here in terms of the damage multiplier of the model. It reflects therefore the amount of bond degradation. Fig. 17 shows the geometry of the EB niche and the calculated damaged zone in terms of contours of equal damage multiplier. It has a null value when the rock is in an undamaged state and a positive value when the rock is damaged. At end of the excavation, the calculated damage zone reaches a thickness of $0.30 \mathrm{~m}$ at the sidewall and $0.60 \mathrm{~m}$ on the niche crown. This is in accordance with some hydraulic and geophysical measurements reported by BGR (2003). They report higher permeability values (up to two orders of magnitude) within a ring of $0.60 \mathrm{~m}$ from tunnel wall. They back calculated the permeability of the host rock based on pulse test and constant head data coming from different boreholes. The sharp lower corner induces a bigger damage zone. Fig. 18 shows the evolution of the damage multiplier at different positions on the sidewall (dash line) and roof (continuous line) of the niche. The damage multiplier increases quickly during the first ten days of drying to increase later at a lower rate. This effect is associated with the change in the suction in the rock. The intensity of damage decreases as the distance to the exposed tunnel wall increases, and it reflects the distribution of suction. However, the shape of the tunnel and the initial state of stress controls the damage pattern around 

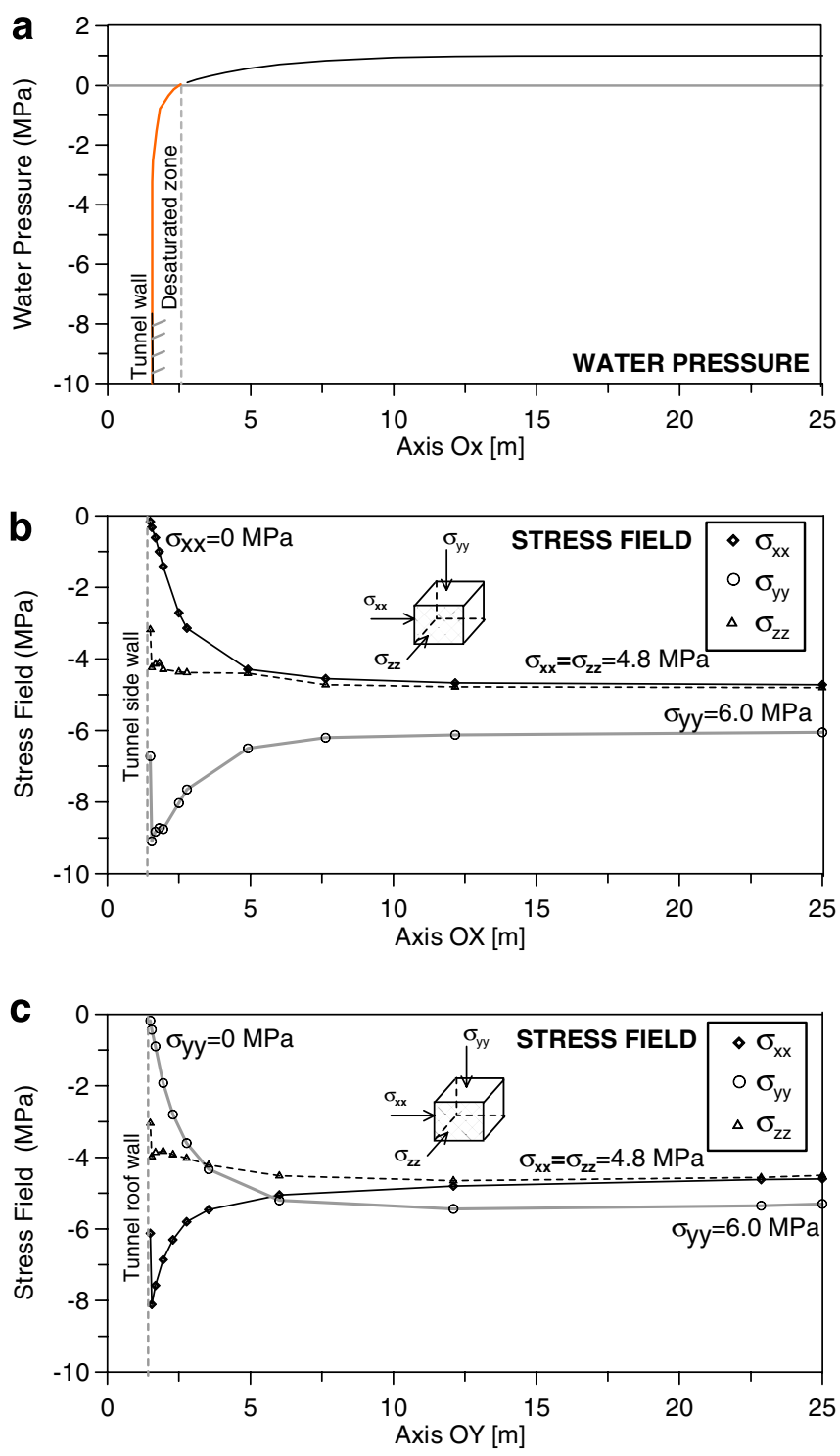

Fig. 13. Variation of water pressure and total stress field along horizontal and vertical axes.

the opening. Results obtained are also consistent with the EDZ characterization reported by Bossart et al. (2002).
They study the EDZ by mapping the frequency of the fractures using overcoring of resin-filled EDZ fractures. They found that the EDZ consists of two zones: an inner zone (close to the tunnel) containing an interconnected fracture network and an outer zone with partially connected or isolated fractures. Maximum thicknesses of the inner zone measured in tunnels excavated by a road header (as in the EB) were 0.60 and $0.90 \mathrm{~m}$ for the tunnel wall and roof, respectively. A thicker EDZ may be defined if the outer zone is included.

\subsection{Buffer hydration phase}

\subsubsection{Wetting history for computational purposes}

After the 160 day ventilation period, buffer materials were emplaced and the hydration phase started.

Since the real scale tests is fully three-dimensional specially if one considers the sequence of hydration, some different hypothesis have to be adopted to reproduce the real hydration history in the 2D model developed. The following characteristics of the hydration system are relevant in this regard:

- Tubes are connected in such a way that the water flows into buffer materials from the floor to the roof and from the front to the end of the excavation.

- Only the history of the total injected water volume and injection pressure at the water reservoir tank were controlled.

- No additional information is available considering the internal distribution of flow rates and water pressures except for the reaction of the different sensors located in the buffer.

The major uncertainty, however, lies in the water losses through the host rock and the concrete plug. Leakages were observed around the plug immediately after the beginning of the injection. If the known injected water volumes are plotted against time (Fig. 19) the accumulated volume exceeds the calculated volume of voids (135001) in July 2003, 450 days after the beginning of the test, when the

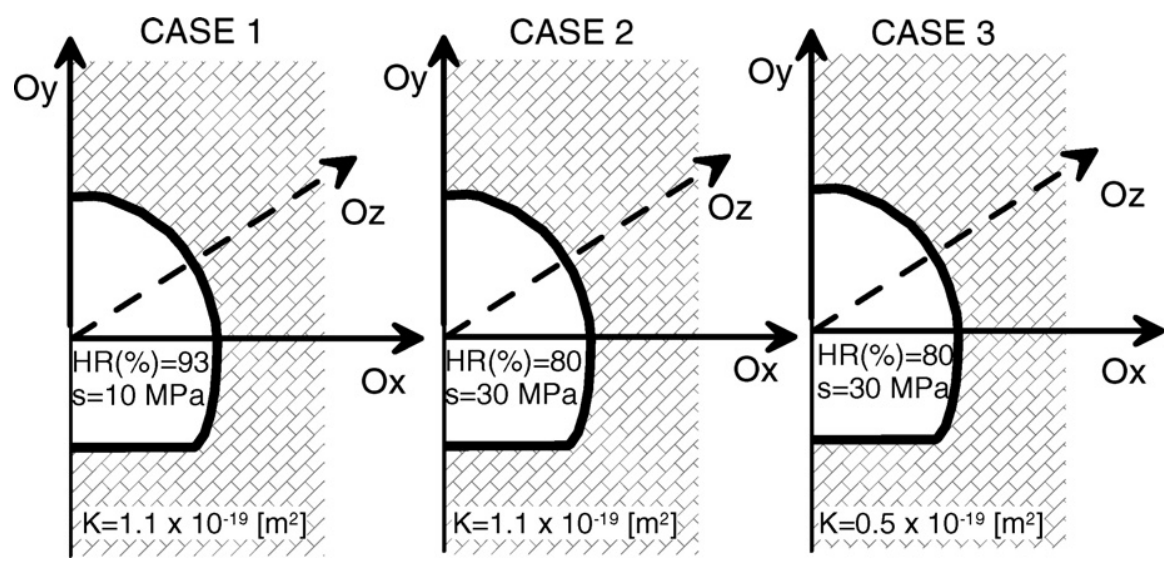

Fig. 14. Rock intrinsic permeability and tunnel relative humidity adopted in calculations. 

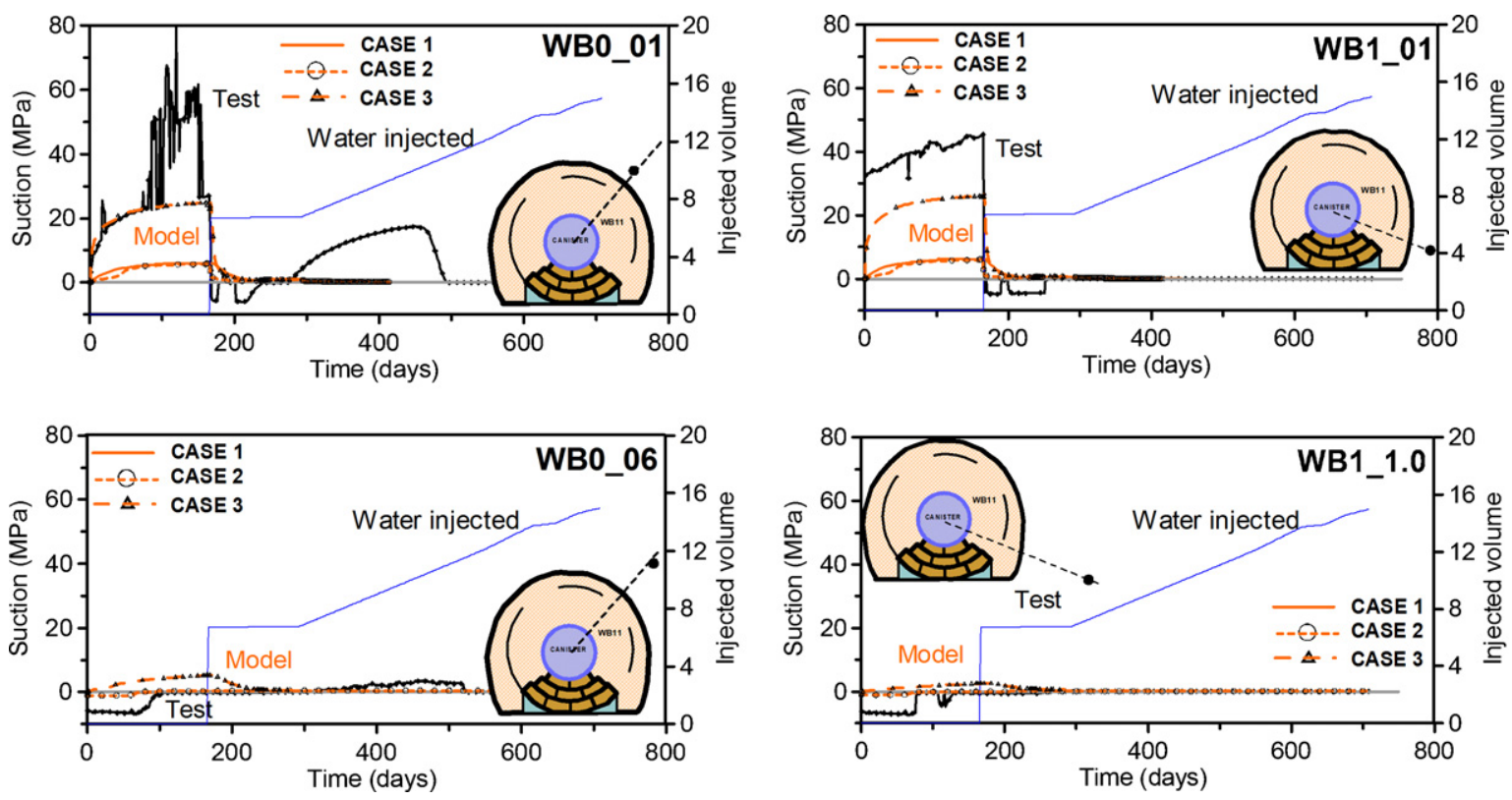

Fig. 15. Evolution of the relative humidity in the rock represented in terms of suction. Model predictions and measured values corresponding to sensors located in section A1.
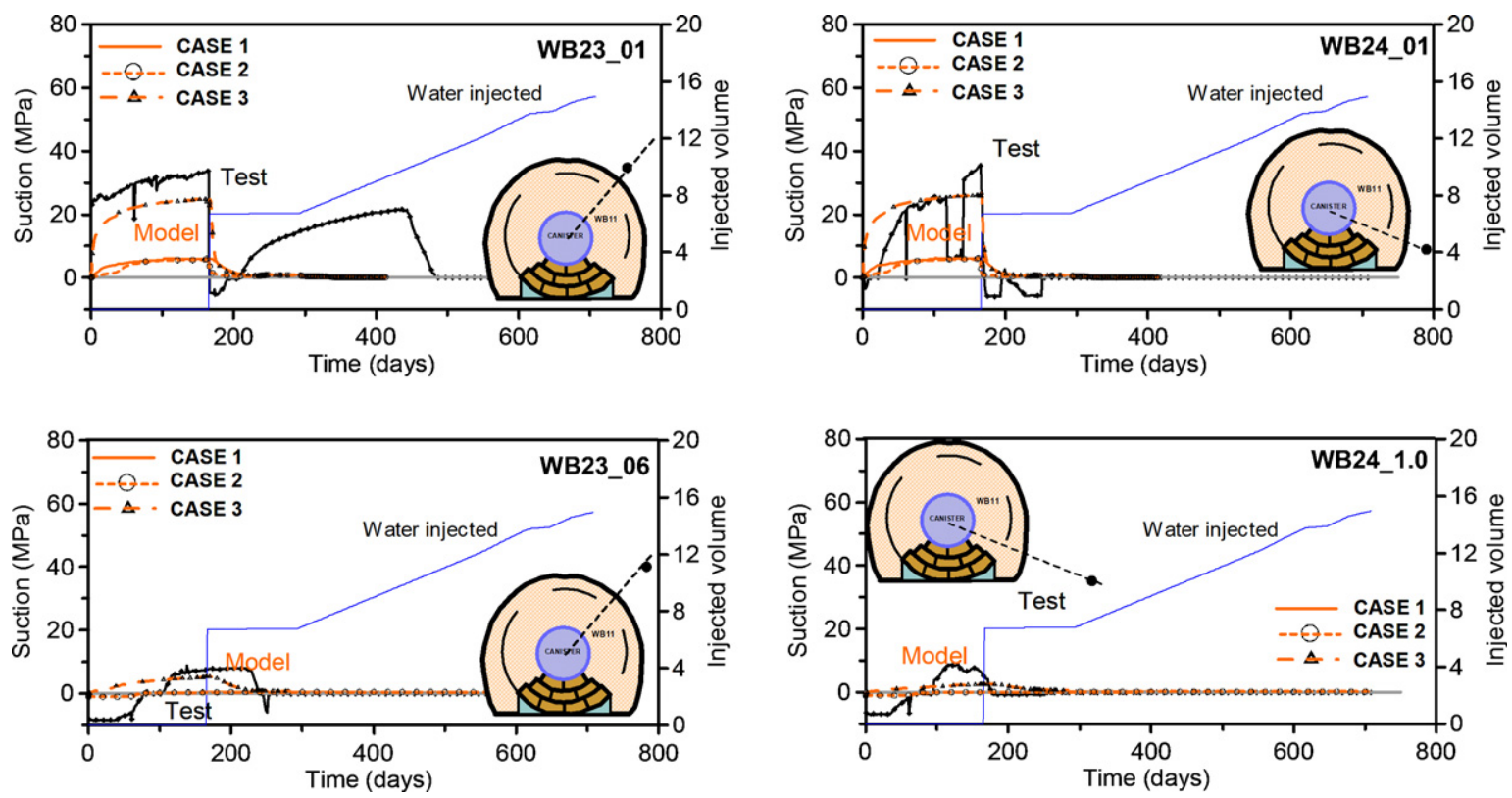

Fig. 16. Evolution of the relative humidity in the rock represented in terms of suction. Model predictions and measured values corresponding to sensors located in section A2.

buffer was far from being saturated. Therefore, no reliable information on the amount of water actually hydrating the buffer is available. On the other hand, the model reacts to imposed flow rates with increments in the water pressure which may not be realistic. In order to approximate the volume of water actually hydrating the buffer, the following sequence of boundary conditions were applied. A constant injection pressure of $10 \mathrm{kPa}$ was assumed during the first 65 days of injection. Then a rest period of 55 days was imposed. Then, on day 120 after the beginning of the injection, a constant injection rate of 201 /day was introduced. However, if the injection pressure increases beyond $10 \mathrm{kPa}$, a constant pressure condition $p_{\mathrm{w}}=10 \mathrm{kPa}$ was imposed at the injection points. The calculated history of water volumes injected is also given in Fig. 19. For the "automatic" water injection period (beyond September $11,2002)$ the model predicts a decrease in water injection rate. The constant rate of water actually injected is not considered a realistic rate for the water actually hydrating the buffer, for the reasons given. 


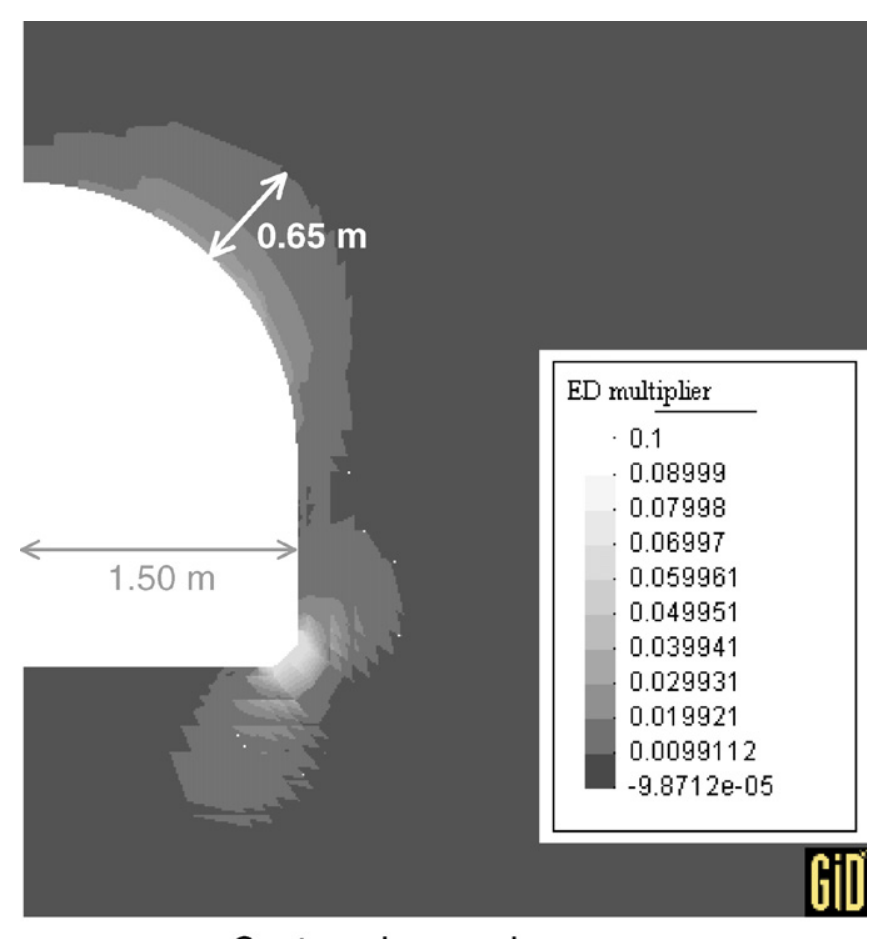

Contour damaged zone

Fig. 17. EDZ computed in terms of damage multiplier after 165 days of tunnel excavation.
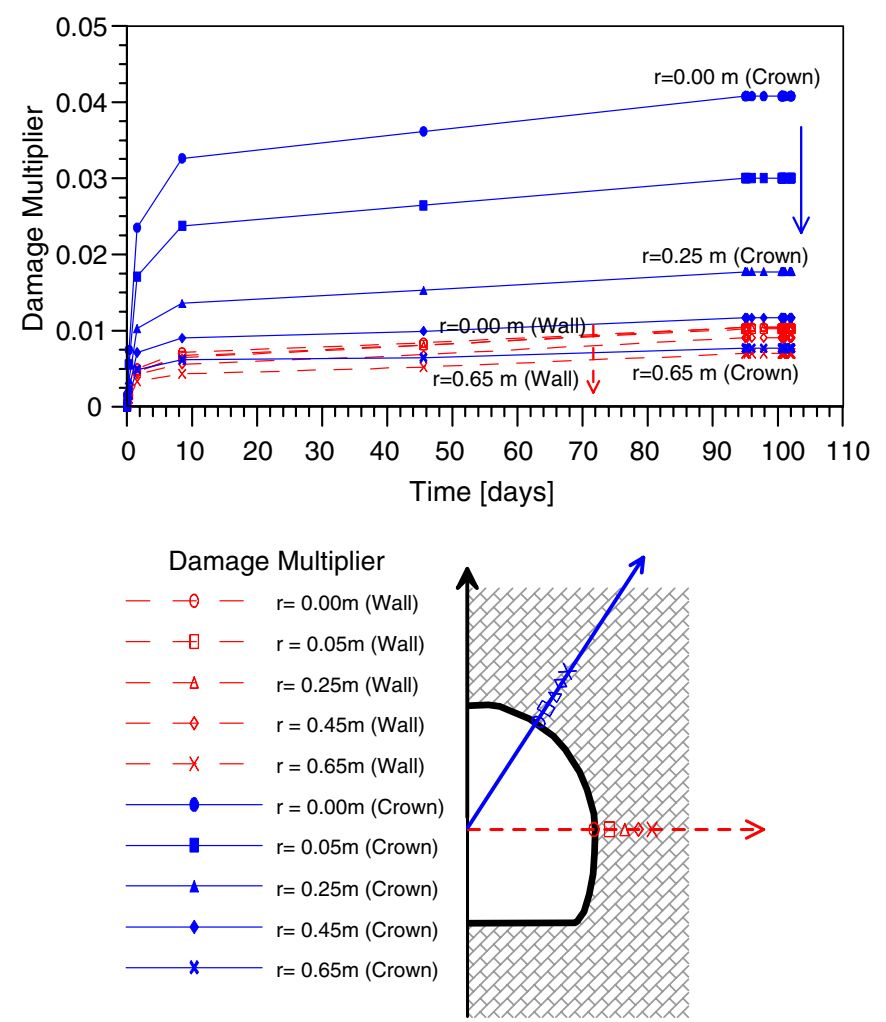

Fig. 18. Evolution of the damage multiplier for different distances to the tunnel surface.

\subsubsection{Buffer behaviour}

Swelling pressure and the relative humidity were monitored in the buffer materials during the hydration phase.

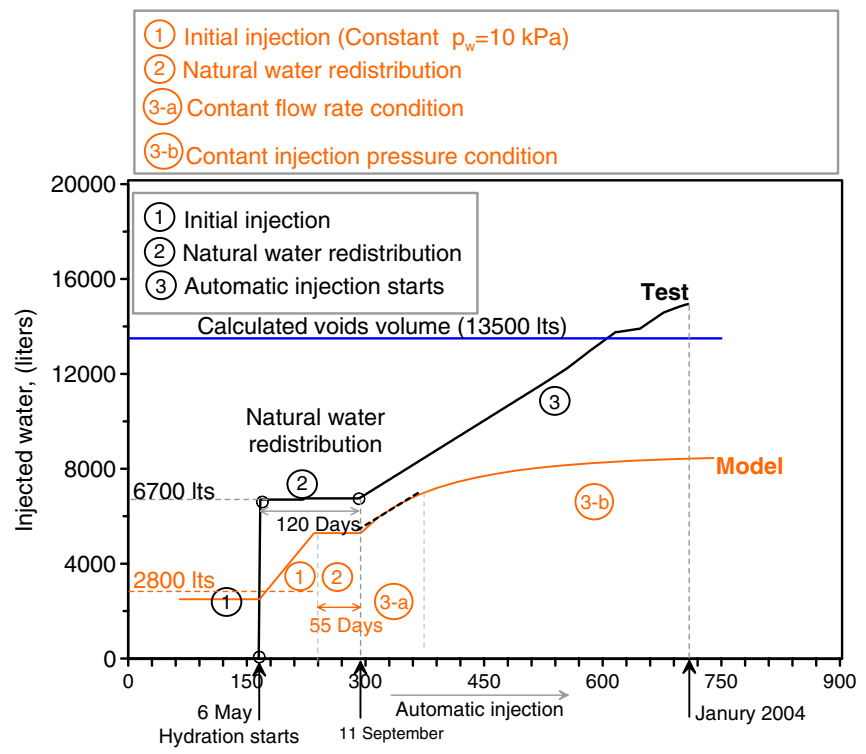

Fig. 19. Total injected water volume and computed volumes actually hydrating the buffer are compared.

Swelling pressure was measured in the central section of the tunnel (section E) and the relative humidity evolution was monitored in two sections (B1 and B2), Fig. 6b and c. Some additional information regarding the movement of the canister was obtained through the extensometers emplaced at both ends of the metallic cylinder, Fig. 6a. In the following paragraphs, comparison between measured and computed results will be presented.

\subsubsection{Relative humidity}

Relative humidity was monitored in two sections by means of four sensors emplaced in each one of them, two located within the blocks and two within the pellets, Fig. 6b. The corresponding suction values were calculated by means of the psychrometric relationship (Eq. (8)). A comparison between the real and computed values is shown in Fig. 20. The history of injected water it is also given in all the comparison plots as a general reference.

The evolution of suction within the bentonite pellets buffer for sections B1 and B2 is presented in Fig. 20a (sensors WB11, WB12, WB21 and WB22). In section B1 (sensors WB11 and WB12) suction decreased fast but in section B2 (sensors WB21 and WB22) a slower wetting is observed. These results provide an idea of the heterogeneous hydration process taking place in the mass of pellets. The model predicts a fast decrease in suction due to the relative large volume of water initially injected. However the RH measurements show a slower hydration rate.

In the case of bentonite blocks (sensors WB13, WB14, WB23 and WB24) Fig. 20b, a more consistent picture emerges. The water redistribution state is reflected in the sensors response. The final injection stage, which provided a constant daily volume of water results in an increased 

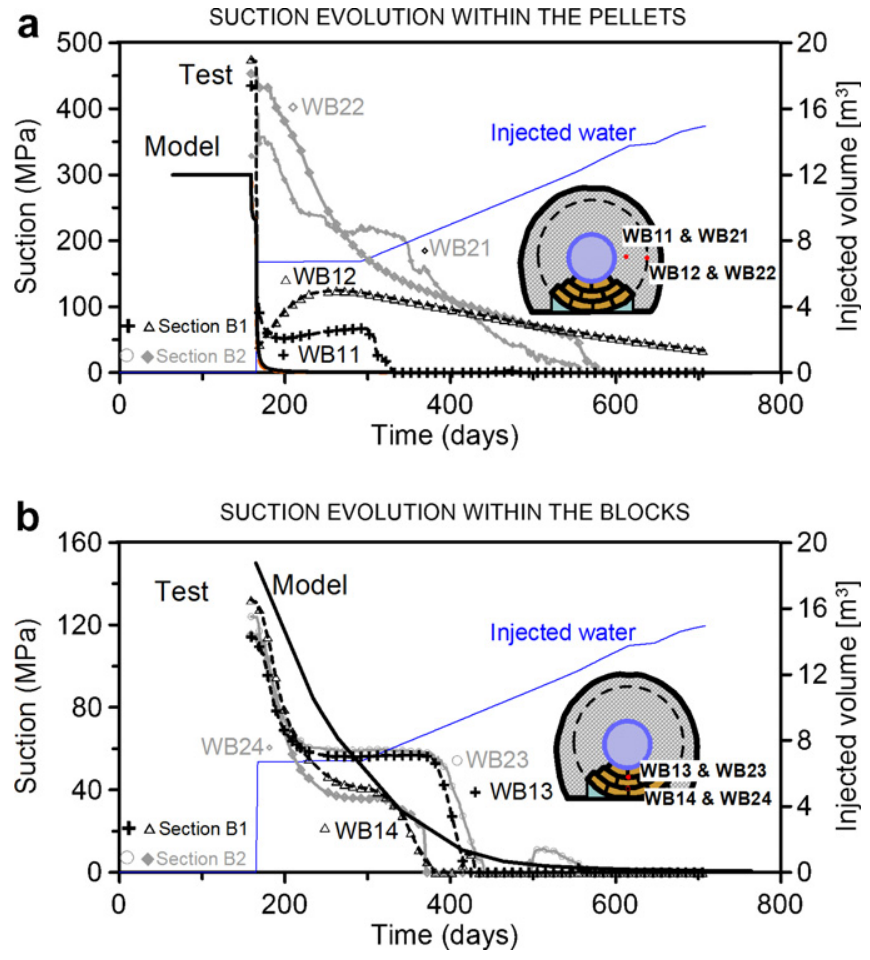

Fig. 20. Evolution of suction during the hydration phase. Measured and computed values are presented for: (a) Sensors located within the bentonite pellets (WB11, WB12, WB12 and WB22); (b) Sensors located within the bentonite blocks (WB13, WB14, WB23 and WB 24).

rate of suction reduction. The calculated response of the blocks is reasonably well predicted although the intermediate redistribution period is barely noticed in the model. The bentonite blocks seem to be close to saturation after 400 days of hydration. Some areas within the pellet buffer remain partially saturated after 600 days of hydration.

\subsubsection{Swelling pressure}

Swelling pressure was monitored by means of eight total pressure cells located in the central section of the tunnel (sensors PE1-PE8 located in section E), Fig. 6c. Measured and computed values corresponding to the pellet buffer material are presented in Figs. 21 and 22. Good agreement is found between computed and predicted stress evolution especially for the vertical stress sensors (PE1 and PE2). The swelling pressure evolution observed in sensors PE1, PE3 and PE7 shows some transient pressure drops which have been associated with local collapse phenomena of the granular fill. However, these observed effects could not be reproduced in the numerical simulations despite the nature of constitutive model used for the bentonite pellets, especially well suited to capture collapse.

Measured and computed results for the swelling pressure of bentonite blocks are shown in Fig. 23. Results obtained in the in situ test are well reproduced by the numerical model. No drops in the swelling pressure evolution are observed in the bentonite blocks, which is probably a result of its high-density.
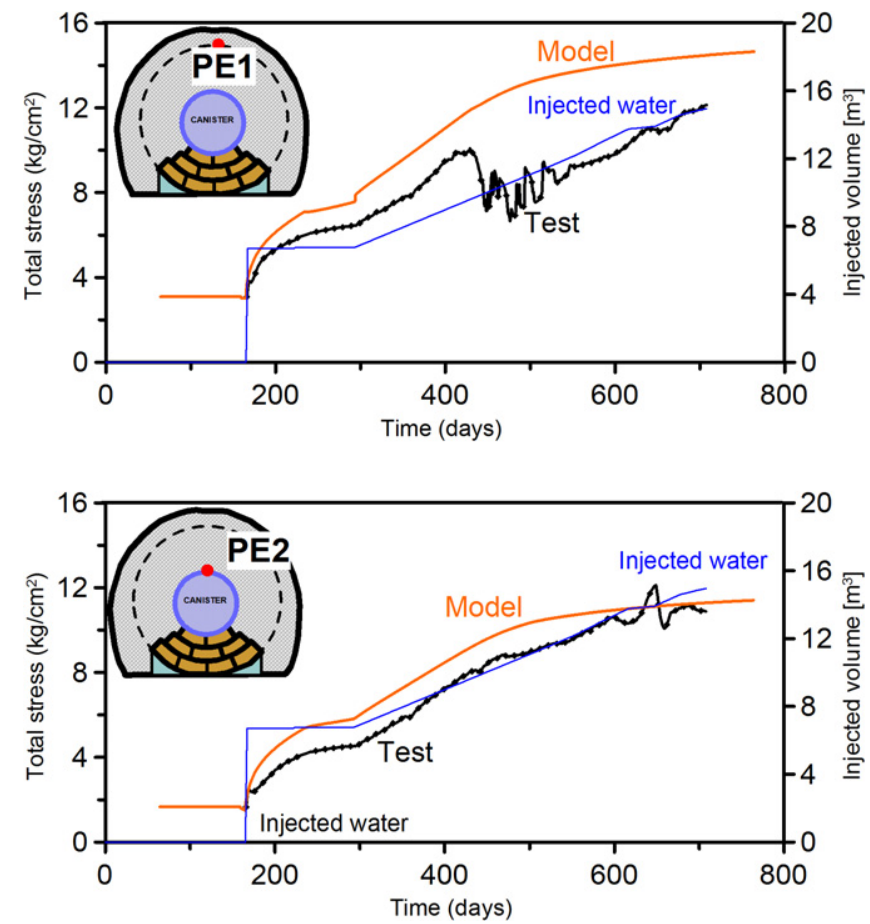

Fig. 21. Calculated and measured swelling pressure during the hydration phase for sensors located within the pellets: PE1 and PE2.
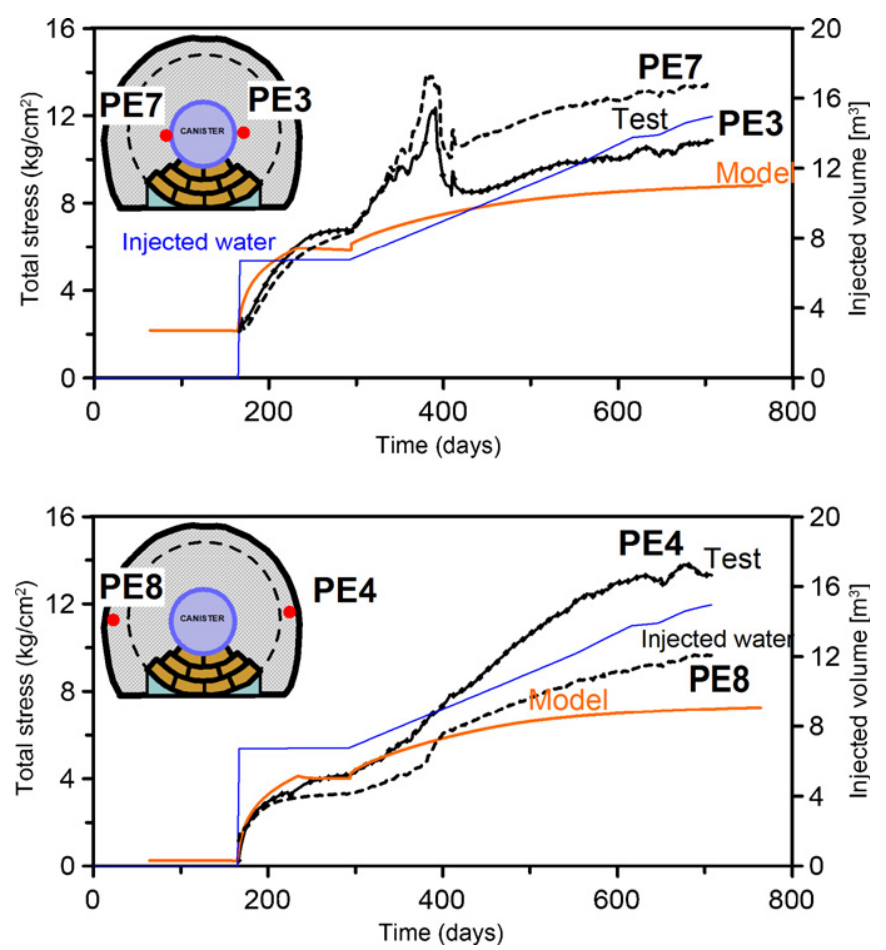

Fig. 22. Calculated and measured swelling pressure during the hydration phase for sensors located within the bentonite pellets: PE3, PE4, PE7 and PE8.

\subsubsection{Movement of the canister}

The movement of the canister was monitored by means of two extensometers emplaced in sections corresponding 

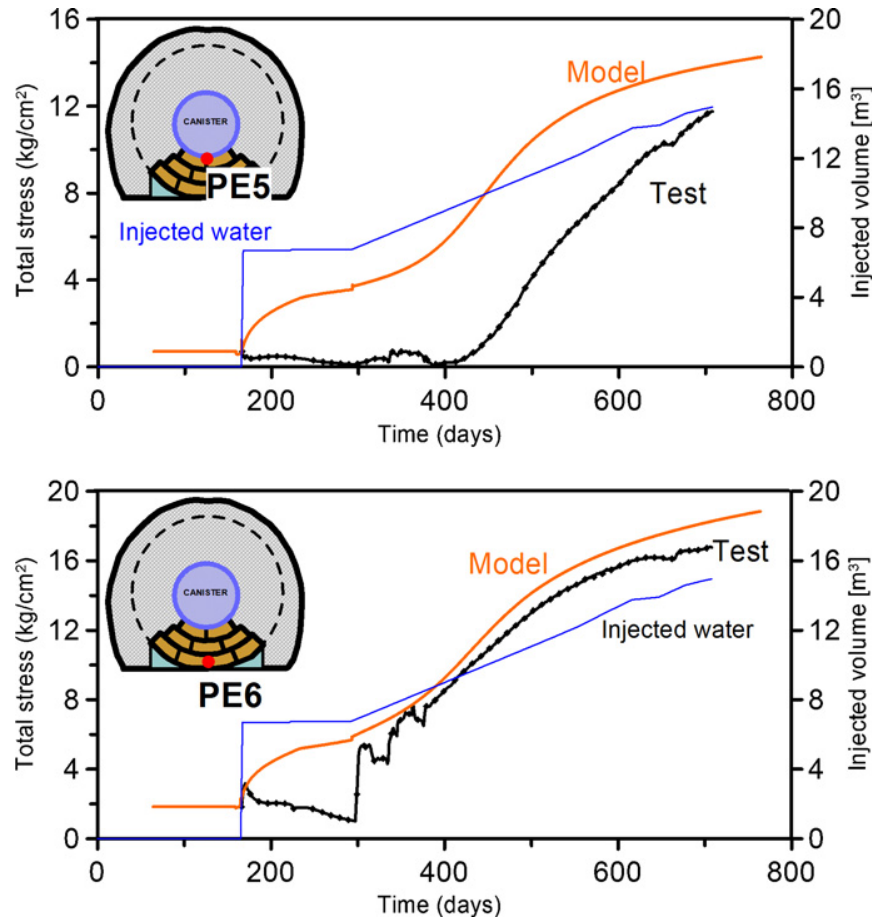

Fig. 23. Calculated and measured swelling pressure during the hydration phase for sensors located within the bentonite blocks: PE5 and PE6.
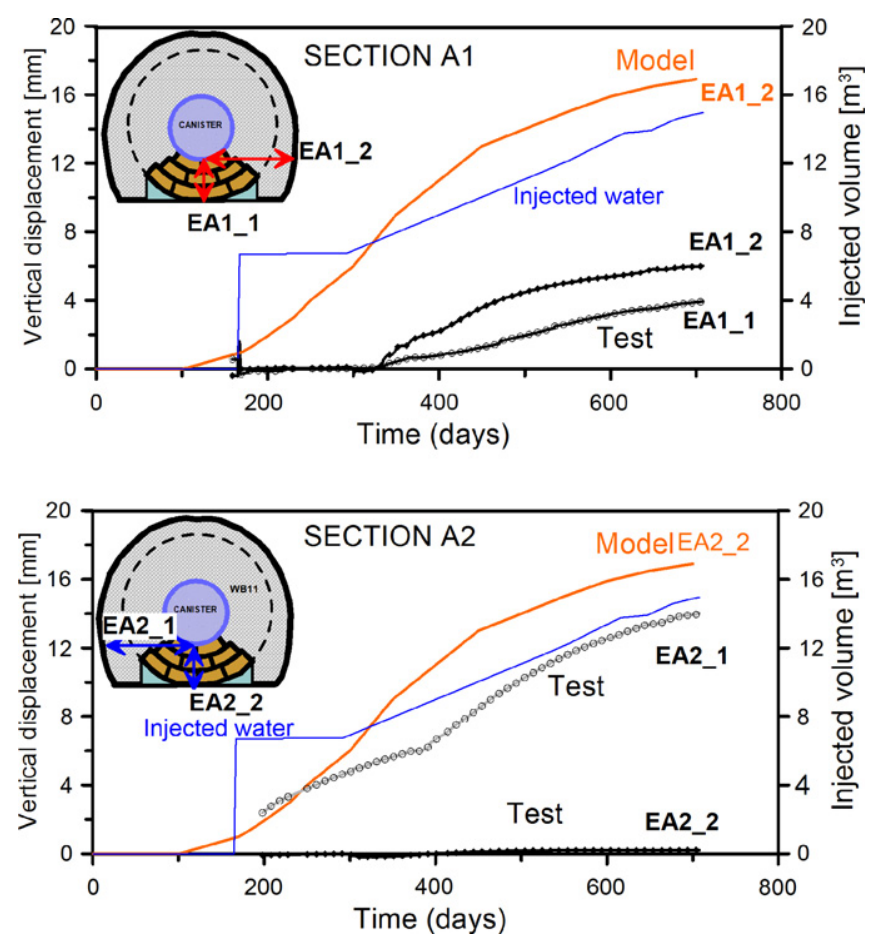

Fig. 24. Calculated and computed displacements of the canister during the hydration phase. A positive measurement in the vertical extensometers will indicate an upward movement of the canister and a positive measurement in the horizontal extensometers is interpreted as a left side movement (considering a frontal view).

at the ends of the canister, sections A1 and A2 in Fig. $6 \mathrm{a}$. The movement of the canister observed during the test is presented in Fig. 24. In section A1 (sensors EA1/1 and
EA1/2) an upward movement of $6 \mathrm{~mm}$ and a left to right horizontal movement of around $4 \mathrm{~mm}$ were measured. In section A2 (sensors EA2/1 and EA2/2), no vertical movement was registered but a right to left displacement of around $14 \mathrm{~mm}$ was measured. Zero horizontal displacement are obviously predicted by the model because of symmetry conditions and an upward movement of $16 \mathrm{~mm}$ was calculated at the end of the modelled period. The reason for this calculated upward motion is the higher swelling potential of the dense blocks compared with the lighter pellet mixture.

Significant discrepancies are observed when the observed and predicted behaviour are compared especially as far as the horizontal displacement is concerned. Horizontal movement of the canister could be related to the different dry density value of the buffer material at both sides of the canister. The vertical movement is probably caused by the higher dry density value of the bentonite blocks with respect to the bentonite pellets. This upward tendency is qualitative reproduced by the model, but not the actual displacement.

\section{Discussion and conclusions}

The engineered barrier (EB) experiment was performed at the underground laboratory of Mont Terri, Switzerland. The paper provides details about the materials characteristics, emplacement procedure, the artificial hydration system and the field instrumentation. A 2D analysis of the problem considering a central plane of symmetry, which follows the sequence of test installation and hydration, was performed. Special attention was given to the selection of the constitutive laws and parameters to represent the two bentonite buffer materials and the host media (Opalinus clay). In the case of the pellet mixture, parameters were derived from the set of laboratory experiments reported in a companion paper (Hoffmann et al., 2006). Parameters for the compacted bentonite blocks and the host rock were taken from previously available information. Results obtained are presented and compared with the actual field behaviour. The following results are highlighted:

- A damage model for the rock was included in the analysis. Because of the tunnel opening, a damaged zone (EDZ) was induced around the excavation. The calculated thickness of the EDZ is similar to in situ determinations based on geophysical procedures and local determinations of permeability (BGR, 2003).

- The desaturation/saturation response of the rock and the associated damage is critically controlled by boundary conditions at the tunnel and by the rock water retention properties and permeability. Some uncertainties remain on the exact values actually operating in the EB experiment.

- The evolution of Relative Humidity in the bentonite blocks which constitute the "cradle" of the simulated canister reasonably well captured by the model. How- 

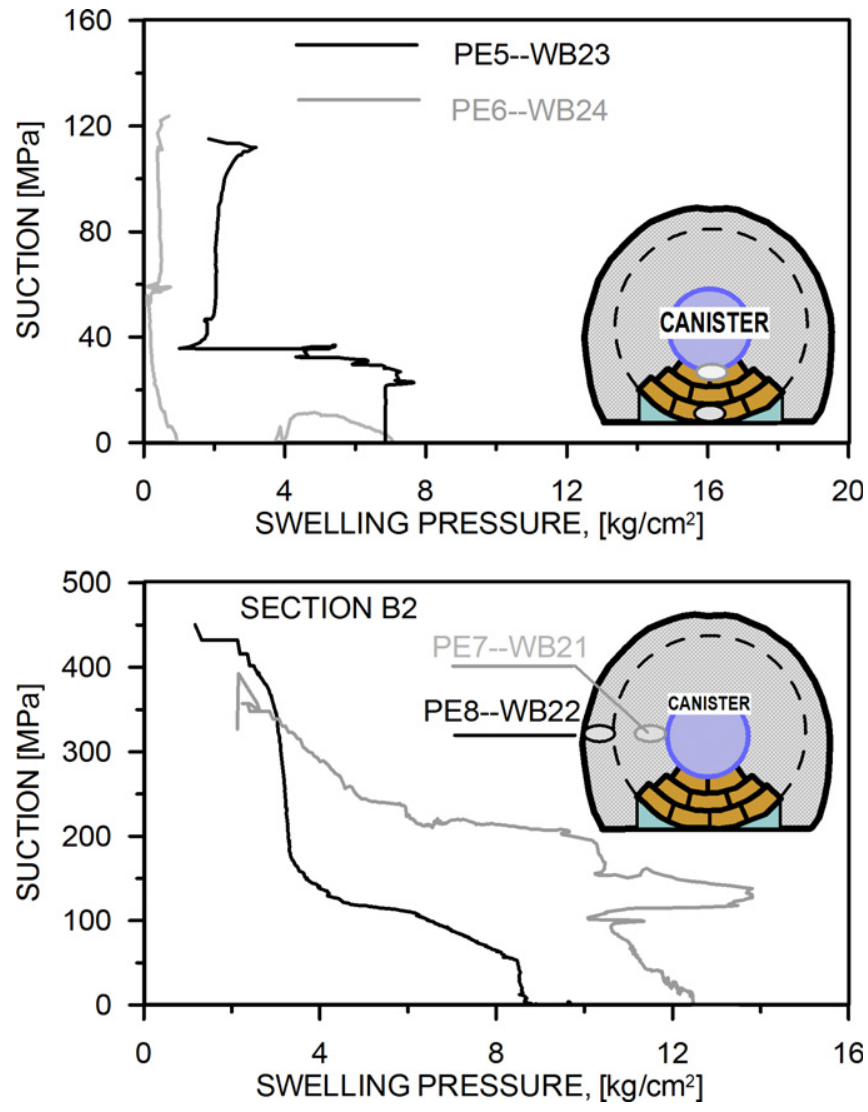

Fig. 25. In situ stress paths (suction against swelling pressure) measured within bentonite blocks and pellets.

ever, calculated hydration rates in the pellet fill are faster than measurements. This is attributed to the complex evolution of permeability of the pellet mixture as it becomes wetted.

- Measured total stresses within the bentonite blocks are well reproduced by the model. A good agreement was also found for stresses in the pellet buffer, specially for the vertical components. Given the strong confinement imposed by the host rock, points within the buffer experience a "swelling pressure" type of path as they become hydrated and at the same time, they develop a swelling strain. Stress paths of this nature can be plotted for points in which total stress cells and relative humidity sensors were available. They are given in Fig. 25 for two points located within the blocks and two points located within the pellets. The irregularities shown by the plotted paths indicate the heterogeneity of the buffer. Clear collapse behaviour is observed in points located within the pellets fill.

- Small but measurable vertical and horizontal displacements of the canister have been recorded. The unexpected horizontal displacement reflects the heterogeneous nature of the pellets fill, or the non-uniform hydration method. The model overestimated the actual upward vertical displacement of the canister. Measured maximum vertical displacement $(14 \mathrm{~mm})$ are however close to the maximum calculated values $(17 \mathrm{~mm})$.

\section{Acknowledgements}

The work described has been supported by ENRESA through the 'Engineered barrier emplacement experiment in Opalinus clay (EB experiment)' (2000-2003). The authors also acknowledge the financial support provided by the EC under the contract FIKW-CT-2000-00017.

\section{References}

AITEMIN, 2001. The EB Experiment engineered barrier emplacement in Opalinus clay. Test Plan, version 3.0.

Alonso, E.E., Gens, A., Josa, A., 1990. A constitutive model for partially saturated soils. Géotechnique 40 (3), 405-430.

BGR, 2003. Engineered barrier emplacement in Opalinus clay. Hydrogeological Characterization of the EDZ. Deliverable 18 of the project.

Bossart, P., Meier, P.M., Moeri, A., Trick, T., Mayor, J.C., 2002. Geological and hydraulic characterization of the excavating disturbed zone in the Opalinus clay of the Mont Terri rock laboratory. Engineering Geology 66, 19-38.

Carol, I., Rizzi, E., Willam, K., 2001. On the formulation of anisotropic elastic degradation. I. Theory based on a pseudo-logarithmic damage tensor rate. International Journal of Solids and Structures. 38, 491518.

ENRESA 2000. FEBEX Project. Full scale engineered barriers experiment for a deep geological repository for high level radioactive waste in crystalline host rock. Final Report. Madrid.

Hoffmann, C., Alonso, E.E., Romero, E. 2006. Hydro-mechanical behaviour of bentonite pellets mixtures. In: Second International Meeting on Clays and Natural and Engineered Barriers for Radioactive Waste Confinement, Tours, France.

Muñoz, J., Alonso, E.E., Vaunat, J., 2004. Development of damaged zones in the vicinity of the barrier-rock interface. In: Proceedings of the European Commission CLUSTER Conference and Workshop on EDZ in Radioactive Waste Geological Repositories, Luxembourg, Nuclear science and technology, EUR 21028 EN, pp. 201-206.

NAGRA. 2003. Engineer barrier emplacement experiment in Opalinus clay (EB). Granular Material Emplacement QA Report with Emplacement Description. Deliverable D12 of the EB Project.

Pearson, F.J., Arcos, D., Bath, A., Boisson, J.-Y., Fernández, A.M., Gaebler, H.E., Gaucher, E., Gautschi, A., Griffault, L., Hernan, P., Waber, H.N. 2003. Mont Terri Project - Geochemistry of water in the Opalinus clay formation. Reports of the Swiss Federal Office for Water and Geology, Geology Series, No. 5, Bern, Switzerland, pp. 319.

Vaunat, J., Gens, A. 2003. Bond degradation and irreversible strains in soft argillaceous rock. In: Cullighan, P.J., Einstein, H.H. \& Whittle, A. (Eds.) "Proceedings of Twelfth Panamerican Conference on Soil Mechanics and Geotechnical Engineering", 23-25th June 2003, Boston, United States, Pub. VGE, ISBN 3-7739-5985-0, vol. 1, pp. 479-484. 